\title{
The impact of a limited budget on the corrective action taking process
}

\author{
Jie Songa,b, Annelies Martens ${ }^{\mathrm{a}}$, Mario Vanhoucke ${ }^{\mathrm{a}, \mathrm{b}, \mathrm{c}, \mathrm{d}, *}$ \\ ${ }^{a}$ Faculty of Economics and Business Administration, Ghent University, Tweekerkenstraat 2, 9000 Gent \\ (Belgium) \\ ${ }^{b}$ School of management, Northwestern Polytechnical University, Xi'an, 710129, Shaanxi, (PR China) \\ ${ }^{c}$ Technology and Operations Management, Vlerick Business School, Reep 1, 9000 Gent (Belgium) \\ ${ }^{d}$ UCL School of Management, University College London, 1 Canada Square, London E14 5AA (United \\ Kingdom)
}

\begin{abstract}
The main goal of project control is to identify the deviations between the baseline schedule and the actual progress of the project by measuring the project performance in progress and using the project control methodologies to generate warning signals that act as triggers for corrective actions to bring the project back on track. To that purpose, tolerance limits are set on the required project performance, such that if the warning signals exceed these limits, they should result in appropriate corrective actions.

In this paper, the Earned Value Management (EVM) control method and its extensions are used to test their abilities in taking corrective actions under a budget constraint. More precisely, four different approaches are proposed for allocating the limited budget along the different project phases, and whether a proper allocation of the budget results in an increase of the expected project outcome is measured. A large computational experiment is conducted on a set of artificial projects to assess the efficiency and effectiveness of the budget allocation models. Results show that simply allocating budget according to the time accrue of projects performs better than methods that take cost, time/cost or risk information into account. Moreover, results indicate that allocating a budget that increases in later stages of the project is beneficial for the outcome.
\end{abstract}

Keywords: Project Management; Project Control; Earned Value Management; Budget Allocation; Simulation

\footnotetext{
${ }^{*}$ Corresponding author

Email addresses: jieson.song@ugent.be (Jie Song), annelies.martens@ugent.be (Annelies Martens), mario.vanhoucke@ugent. be (Mario Vanhoucke)
} 


\section{Introduction}

Baseline scheduling, risk analysis and project control are three crucial aspects throughout the project life cycle. The baseline schedule serves as a point-of-reference for risk analysis and project control. Schedule risk analysis is a simulation tool and technique to indicate the sensitive components of the baseline schedule that potentially have a significant impact on improving the accuracy of schedule and reliability of response during project control. The main purpose of project control is to detect potential problems or opportunities by comparing the project progress to the baseline schedule, such that corrective actions will be taken when necessary. The integration of these three phases to control projects is known as dynamic scheduling or integrated project management and control (Vanhoucke, 2012b).

Our main focus of this study lies on the project control systems, which monitor the project performance in progress and control the expected outcome by taking corrective actions. During the monitoring phase, the project manager should make full use of all available information to measure the project performance in progress and set tolerance limits on these measured performance periodically. Once the performance falls below these limits, the project performance is no longer acceptable. During the control phase, the project manager should drilldown to lower levels of the work breakdown structure (WBS) to explore the activities which are responsible for the problems and take corrective actions to bring the expected project outcome back on track. However, the corrective actions always directly result in additional costs (reassignment of highly-skilled personnel, extra equipment, improvement of manpower, etc.) during project progress. Thus, the project manager should set aside additional budget to implement the interventions and the amount of available budget is limited. In order to make the best use of the limited budget, they should be allocated in an efficient and effective way to maximize the project outcome.

In this paper, four different approaches are proposed to allocate the limited budget to take corrective actions with the aim of improving the expected project outcome. More precisely, three of these four approaches make full use of the dynamic information on project work completion obtained from earned value metrics (EV), earned schedule metrics (ES) and earned duration management metrics (EDM) to allocate the limited availability of budget, while the fourth one follows the static information on the sensitivity of individual activities retrieved from the schedule risk analysis (SRA) prior to the project execution to model allocating budget.

The structure of this paper is described as follows. In Section 2, a literature review on project control in general and the corrective action taking process in particular is presented. In Section 3, the four approaches to allocate control budget to take corrective actions and the design of the experiments are clarified. Section 4 explicitly discusses the computational 
results of the simulation study. The overall conclusions of the computational experiment and future research are provided in Section 5 .

\section{Problem formulation}

This section gives a detailed literature review on project control with corrective actions and shows where the current study fits in the existing research. More specifically, Section 2.1 summarizes the existing literature and discusses 34 studies using four criteria relevant to our study. Based on the literature review, Section 2.2 gives an overview of the specific problem studied in the current paper.

\subsection{Literature review}

The research on controlling projects with Earned Value Management has grown in the last decades, and the topic has been investigated from different angles. Many of these research studies make use of so-called tolerance limits in different ways. The central idea is that the project performance is measured during the project progress and is compared to these predefined tolerance limits. Once the tolerance limits have been exceeded, the current project progress is no longer acceptable, and corrective actions are then necessary to bring the project back to an acceptable status. Hence, tolerance limits serve as warning signals for taking corrective actions to bring projects in danger back on track. Table 1 summarizes the relevant research studies on project control using tolerance limits, and the studies are divided using four criteria relevant to our study, which will be described along the following paragraphs.

Performance measurement: Monitoring the performance of a project in progress can be done by monitoring the performance of each individual activity of the project, or can be done by monitoring a set of activities (e.g. work packages) simultaneously without looking at the status of each individual activity. The table shows that 7 studies measure the performance of individual activity of the project (column "Activity"), while 29 studies focus on a higher level of the work breakdown structure (column "Project"). These project-based performance studies make use of the schedule and cost performance indices that serve as general project performance metrics for a set of activities, rather than measuring the progress of each activity individually. In Lipke et al. (2009), it has been argued that such a project-based control approach is the only practical way of monitoring the project progress, and Table 1 indeed shows that most studies focus on measuring the progress at the project level. Only a handful of studies measure the progress at the individual activity level, and some authors like Book (2006) state that controlling individual activity always leads to better results. In a simulation experiment conducted by Vanhoucke and Vandevoorde (2007), it is measured what the impact is of a project-based control approach compared to an activity-based approach, and 
the authors conclude that EVM is often a reliable tool for measuring project delays when used at the project level rather than on the activity level.

Tolerance limits: The EVM data collected during the project's progress to measure its performance can be used to set thresholds for taking corrective actions. The literature on using tolerance limits for project control has grown significantly in the last few years, and has been classified in three types on approach in Martens and Vanhoucke (2017a). The static tolerance limits are the easiest since they only make use of fixed thresholds using the EVM data. They do not require much calculations or simulations since they simply set minimum or maximum values for the schedule performance index (SPI) or the cost performance index (CPI). Consequently, these static limits are nothing more than pragmatic rules-of-thumb and often make use of fixed values (e.g. the performance cannot drop below the $90 \%$ performance) used for all projects in the company's project portfolio. The analytical tolerance limits go one step further, and require some additional analyses before thresholds can be set. More specifically, the thresholds are now set based on some project characteristics rather than using arbitrary numbers, and therefore, each project can have different values of the thresholds at different phases of its progress. Nevertheless, these analytical thresholds are still rather easy to use since they do not require advanced simulations or statistical analyses. The statistical tolerance limits are the most advanced tolerance limits since they require the use of more advanced statistical analyses and some pre-analysis of the specific project using Monte Carlo simulations. With the growing availability of project data and the ease of use of simulation software tools, Table 1 shows that 18 studies make use of these statistical tolerance limits. Nevertheless, Vanhoucke (2018) argues that the use of analytical tolerance limits is also a promising research direction, since they often perform almost as good as the statistical tolerance limits, but are much easier to use in a practical setting. Table 1 shows that 10 studies are available for these easy-to-use analytical tolerance limits.

Corrective actions: Obviously, the use of tolerance limits is only relevant when they are used as warning signals for taking corrective actions. Some research studies only focus on providing a detailed methodology on how such tolerance limits should be constructed to predict project problems in advance. However, other studies do not only focus on the tolerance limits calculations, but also measure whether they can be used for taking corrective actions. Such studies measure the impact of actions on the project performance, rather than restricting their focus on measuring the reliability of tolerance limits to predict project problems. Table 1 shows that the majority of the studies ignore corrective actions, but nevertheless 11 studies incorporate them in their research study. Most of these studies consider the use of activity crashing as the only possible corrective action. In this view, an action is defined as a reduction in the activity duration at the expense of a growing cost. Recently, Madadi and Iranmanesh (2012) have extended this option to variability reduction which considers action as a way to reduce the uncertainty in the activity duration. 
Control restrictions: Some studies have extended the research on tolerance limits by adding additional restrictions for the corrective actions. Such additional restrictions assure that actions cannot be simply taken without considering other side effects such as limited budgets or other limitations. Table 1 shows that only 3 studies have set limits on possible set of actions taken during project control, and each study has defined these restrictions in a different way. Hegazy and Petzold (2003) presents a practical model to determine optimized activity crashing during project control, and they take time, cost and resource constraints into account. In the study of Vanhoucke (2010), the number of corrective actions is limited by introducing the concept of control effort. Finally, Martens and Vanhoucke (2019) set limits on corrective actions by specifying a strict budget limit available along the project life cycle. All of these limitations are set to fixed values (column "Fixed") which means that they do not change along the life of the project. In our study, we will explore different versions of variable restrictions which will be discussed in the next section. 
Table 1: Literature list.

\begin{tabular}{|c|c|c|c|c|c|c|c|c|c|c|c|c|}
\hline & \multirow[t]{2}{*}{ References } & \multicolumn{2}{|c|}{ Project performance } & \multicolumn{3}{|c|}{ Tolerance limits } & \multicolumn{3}{|c|}{ Corrective actions } & \multicolumn{3}{|c|}{ Control restrictions } \\
\hline & & Activity & Project & Static & Analytical & Statistical & No & Crashing & Variability & No & Fixed & Variable \\
\hline \multirow{23}{*}{ 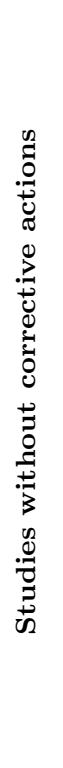 } & Bauch and Chung (2001) & - & $\checkmark$ & - & - & $\checkmark$ & $\checkmark$ & - & - & - & - & - \\
\hline & Leach (2005) & - & $\checkmark$ & $\checkmark$ & - & - & $\checkmark$ & - & - & - & - & - \\
\hline & Barraza and Bueno (2007) & - & $\checkmark$ & - & - & $\checkmark$ & $\checkmark$ & - & - & - & - & - \\
\hline & Leu and Lin (2008) & - & $\checkmark$ & - & - & $\checkmark$ & $\checkmark$ & - & - & - & - & - \\
\hline & Pajares and López-Paredes (2011) & - & $\checkmark$ & - & - & $\checkmark$ & $\checkmark$ & - & - & - & - & - \\
\hline & Aliverdi et al. (2013) & - & $\checkmark$ & - & - & $\checkmark$ & $\checkmark$ & - & - & - & - & - \\
\hline & Acebes et al. (2014) & - & $\checkmark$ & - & - & $\checkmark$ & $\checkmark$ & - & - & - & - & - \\
\hline & Colin and Vanhoucke (2014) & - & $\checkmark$ & - & - & $\checkmark$ & $\checkmark$ & - & - & - & - & - \\
\hline & Kim and Kim (2014) & - & $\checkmark$ & - & - & $\checkmark$ & $\checkmark$ & - & - & - & - & - \\
\hline & Colin et al. (2015) & - & $\checkmark$ & - & - & $\checkmark$ & $\checkmark$ & - & - & - & - & - \\
\hline & Colin and Vanhoucke (2015) & - & $\checkmark$ & - & - & $\checkmark$ & $\checkmark$ & - & - & - & - & - \\
\hline & $\operatorname{Kim}(2015)$ & - & $\checkmark$ & - & - & $\checkmark$ & $\checkmark$ & - & - & - & - & - \\
\hline & Perkusich et al. (2016) & - & $\checkmark$ & - & - & $\checkmark$ & $\checkmark$ & - & - & - & - & - \\
\hline & Hammad et al. (2016) & - & $\checkmark$ & - & $\checkmark$ & - & $\checkmark$ & - & - & - & - & - \\
\hline & Ong et al. (2016) & - & $\checkmark$ & - & $\checkmark$ & - & $\checkmark$ & - & - & - & - & - \\
\hline & Martens and Vanhoucke (2017a) & - & $\checkmark$ & - & $\checkmark$ & - & $\checkmark$ & - & - & - & - & - \\
\hline & Martens and Vanhoucke (2017b) & - & $\checkmark$ & - & $\checkmark$ & - & $\checkmark$ & - & - & - & - & - \\
\hline & Martens and Vanhoucke (2018) & - & $\checkmark$ & - & $\checkmark$ & - & $\checkmark$ & - & - & - & - & - \\
\hline & Zhang et al. (2018) & - & $\checkmark$ & - & $\checkmark$ & - & $\checkmark$ & - & - & - & - & - \\
\hline & Zohoori et al. (2018) & - & $\checkmark$ & - & - & $\checkmark$ & $\checkmark$ & - & - & - & - & - \\
\hline & Ballesteros-Pérez et al. (2019) & $\checkmark$ & - & $\checkmark$ & - & - & $\checkmark$ & - & - & - & - & \\
\hline & Chen et al. (2019) & - & $\checkmark$ & - & - & $\checkmark$ & $\checkmark$ & - & - & - & - & - \\
\hline & Hadian and Rahimifard (2019) & - & $\checkmark$ & - & - & $\checkmark$ & $\checkmark$ & - & - & - & - & - \\
\hline \multirow{8}{*}{ 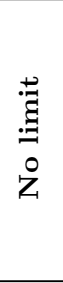 } & Bowman (2006) & $\checkmark$ & - & - & - & $\checkmark$ & - & $\checkmark$ & - & $\checkmark$ & - & - \\
\hline & Vanhoucke (2011) & $\checkmark$ & $\checkmark$ & $\checkmark$ & - & - & - & $\checkmark$ & - & $\checkmark$ & - & - \\
\hline & Madadi and Iranmanesh (2012) & $\checkmark$ & - & $\checkmark$ & - & - & - & - & $\checkmark$ & $\checkmark$ & - & - \\
\hline & Vanhoucke (2012a) & $\checkmark$ & $\checkmark$ & $\checkmark$ & - & - & - & $\checkmark$ & - & $\checkmark$ & - & - \\
\hline & $\mathrm{Hu}$ et al. (2014) & - & $\checkmark$ & - & $\checkmark$ & - & - & $\checkmark$ & - & $\checkmark$ & - & - \\
\hline & Sabeghi et al. (2015) & - & $\checkmark$ & - & - & $\checkmark$ & - & $\checkmark$ & - & $\checkmark$ & - & - \\
\hline & Hu et al. (2016) & - & $\checkmark$ & - & $\checkmark$ & - & - & $\checkmark$ & - & $\checkmark$ & - & - \\
\hline & Ou-Yang and Chen (2019) & - & $\checkmark$ & - & - & $\checkmark$ & - & $\checkmark$ & - & $\checkmark$ & - & - \\
\hline \multirow{5}{*}{ 芞 } & Hegazy and Petzold (2003) & $\checkmark$ & - & - & $\checkmark$ & - & - & $\checkmark$ & - & - & $\checkmark$ & - \\
\hline & Vanhoucke (2010) & $\checkmark$ & - & $\checkmark$ & - & - & - & $\checkmark$ & - & - & $\checkmark$ & - \\
\hline & Martens and Vanhoucke (2019) & - & $\checkmark$ & - & $\checkmark$ & - & - & - & $\checkmark$ & - & $\checkmark$ & - \\
\hline & \# studies & 7 & 29 & 6 & 10 & 18 & 23 & 9 & 2 & 8 & 3 & 0 \\
\hline & Current study & - & $\checkmark$ & - & $\checkmark$ & - & - & $\checkmark$ & - & - & - & $\checkmark$ \\
\hline
\end{tabular}




\subsection{Current study}

This section will outline the problem formulation used in this study, and the last line of Table 1 shows how our study can be compared with the current studies in the literature. It shows that our study makes use of a project-based control system using analytical tolerance limits for taking corrective actions. The corrective action that can be used is the well-known activity crashing option, and each action can be used under variable budget limits.

The problem investigated in the current study makes use of a budget restriction similar to Martens and Vanhoucke (2019). More specifically, the budget is defined as a doublyconstrained resource (Slowinski, 1980) for taking corrective actions, i.e. the budget is constrained in two ways. First, a specific maximum use of budget is set for the whole project, and the cost of all corrective actions made during the project execution cannot exceed this maximum value. Second, each project control stage also has a maximum value on the budget which cannot be exceeded to guarantee that not all corrective actions are taken in one period leaving no money left for later periods in the project progress. The periodic restriction is different in our study compared to the existing studies in literature.

As shown in Table 1, all studies in literature make use of fixed restrictions on the total control budget (Column "Fixed"), which means that each control stage receives a fixed values (e.g. money) regardless of the characteristics of the project and the progress in each stage. The current study extends this fixed approach to variable restrictions which means that the limited budget per period depends on certain project characteristics (Column "Variable"). In this view, some stages receive more budget than others, which gives the project manager for some stages more room for taking corrective actions than others, while he/she still can never exceed the total project budget along the whole life cycle.

The variable periodic budget restriction used in the current study is set in four different ways, each time using some specific characteristics about the current state of the project in progress. A summary of these four methods is briefly discussed along the following lines, and details will be given in Section 3 .

- Earned value approach: The budget allocated to each control phase is set proportional to the values earned at each stage of the project. This approach makes use of the well-known earned value methodology (Fleming and Koppelman, 2010) which calculates project progress in monetary units.

- Earned schedule approach: The budget allocated to each project control phase is defined by a mix of the time and cost progress made at each stage. The somewhat more recently investigated earned schedule methodology (Lipke, 2003) - which can be seen as a time extension of EVM - is used for this budget allocation approach. 
- Earned duration approach: The budget allocated to each control phase is based on the estimated project progress using the earned duration methodology which measures the time progress of a project in progress (Khamooshi and Golafshani, 2014).

- Activity risk approach: The budget allocated to each phase is now depending on the activity risk, i.e. the total risk of all activities that are under control at a particular stage. In doing so, more risky project phases will receive more budget to give the project manager more room for taking actions.

In Section 3.1, the general procedure to model these four budget allocation methods is discussed in more detail. In the remainder of this paper, this limited budget is further referred to as the control budget, since it is used for implementing corrective actions to control the expected project outcome.

\section{Methodology}

In order to investigate the impact of the control budget on the corrective action taking process to improve the expected project outcome, a computational experiment on a large set of projects has been set up. An overview of the design of the experiment is depicted in Fig. 1, which is slightly adapted to the methodology employed in Martens and Vanhoucke (2019). As this figure shows, the experiment is split up into 'definition phase', 'scheduling phase', 'execution and control phase' and 'termination phase'. The main differences with the methodology of Martens and Vanhoucke (2019) are situated in the execution and control phase. First, in the definition phase, a set of projects are generated to provide information on the precedence relations and baseline estimates of activity durations. During the scheduling phase, the baseline schedule is constructed to determine the planned duration and cost for each project, and the project deadline is set by adding a project buffer to the end of the baseline schedule. Moreover, tolerance limits are set up using specific information from the baseline schedule to provide warning signals when the project deadline is expected to be delayed. In the next phase, the execution and control phase, each project is executed dynamically with tracking periods (tp) until the project is completed. Subsequently, the control budget is allocated to each tracking period to support the project manager to take corrective actions when needed. The project progress is measured and compared with the corresponding tolerance limits at each tracking period. When the periodic performance is below these tolerance limits, the project manager should determine whether corrective actions are taken or not. When the allocated budget is sufficient, corrective actions are taken to bring the project back on track. Finally, during the termination phase, the project performance of each project is measured and evaluated in terms of control efficiency and control effectiveness.

Section 3.1 introduces four different control budget allocation approaches. In Section 3.2, the 
general procedure to take corrective actions is discussed. Section 3.3 describes the specific parameter settings used in the simulation experiments. The performance metrics to measure the impact of control budget on corrective actions are proposed in Section 3.4.

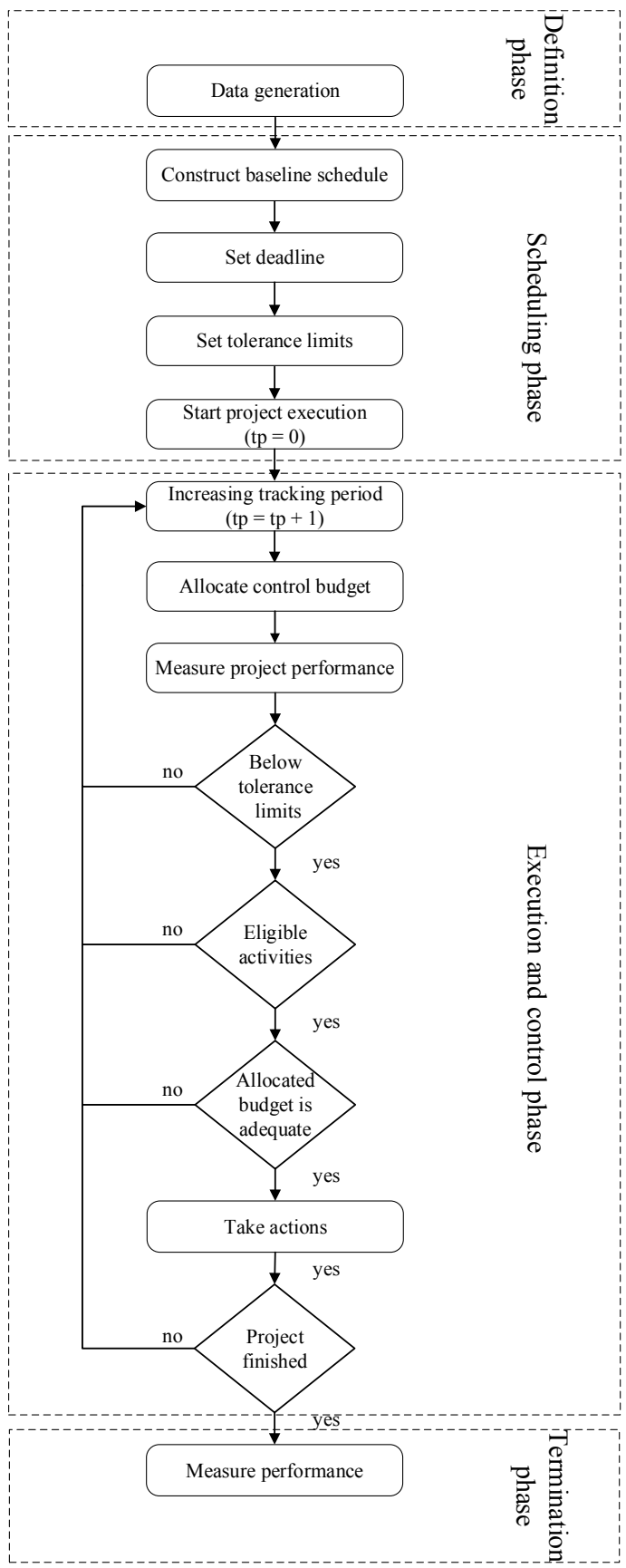

Figure 1: The design of experiment. 


\subsection{Control budget allocation}

In this section, the control budget allocation process is introduced. First, Section 3.1.1 describes the four different approaches to allocate the control budget proportionally to different project phases. Subsequently, Section 3.1.2 extends this procedure by allocating the control budget according to the four approaches in a dynamically increasing or decreasing way. Finally, an illustrative example is provided in Section 3.1.3.

\subsubsection{Allocation}

This section focuses on the construction of the control budget allocation model to determine the allowable budget consumption at each project control phase. The allowable budget consumption defines the maximum portion of the control budget that is allowed to be consumed at each control phase. More specifically, the allowable budget consumption at each tracking period $\left(A B_{t p}\right)$ is determined based on its expected percentage completion $(\mathrm{PC})$ of the next tracking period $(t p+1)$. In doing so, the $A B_{t p}$ can be used to take corrective actions in case the problems occur between $t p$ and $t p+1$, which can be calculated as

$$
A B_{t p}=\left(P C_{t p+1}-P C_{t p}\right) \times \text { control budget }
$$

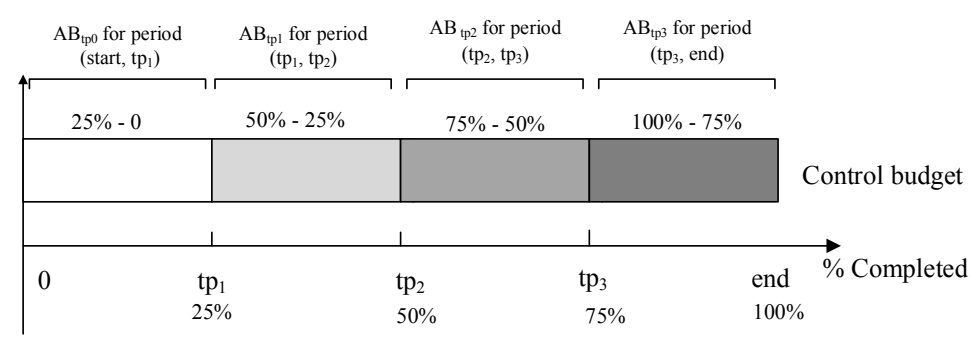

Figure 2: The general idea of determination of $A B_{t p}$.

In order to illustrate how the allowable budget consumption is determined in general, Fig. 2 displays the determination of the $A B_{t p}$ according to the percentage completion of the project. The $\mathrm{x}$-axis denotes the $\mathrm{PC}$ of a fictitious project along the project life cycle, the box above the x-axis represents the $A B_{t p}$. As an example, since at $t p_{1}, 25 \%$ of the project is completed and $50 \%$ of the project is completed at $t p_{2}$, the PC between $t p_{1}$ and $t p_{2}$ is $25 \%$ (= $\left.P C_{t p 2}-P C_{t p 1}=50 \%-25 \%\right)$. Consequently, the $A B_{t p 1}$ is determined as $25 \%$ of the control budget. In the remainder of this section, four specific novel control budget allocation models are proposed based on different ways of calculating the project PC, leading to a different 
portion of allowable budget consumption for each control phase. The abbreviations used for allocating the control budget are summarized in Table 2 .

Table 2: Abbreviations used for control budget allocation.

\begin{tabular}{ll}
\hline Abbreviations & Explanations \\
\hline$A B_{t p}$ & Allowable budget at tracking period tp \\
$P C_{t p}$ & Percentage completion of the project at tracking period tp \\
$E V_{t p}$ & Earned value of the project at tracking period tp \\
$E S_{t p}$ & Earned schedule of the project at tracking period tp \\
$E D_{t p}$ & Earned duration of the project at tracking period tp \\
$A R_{t p}$ & Activity risk of the project at tracking period tp \\
$S S I_{i}$ & SSI value of activity i \\
$T A R$ & Total activity risk value of the project \\
$s_{i}$ & Planned start time of each activity \\
$d_{i}$ & Planned duration of each activity \\
\hline
\end{tabular}
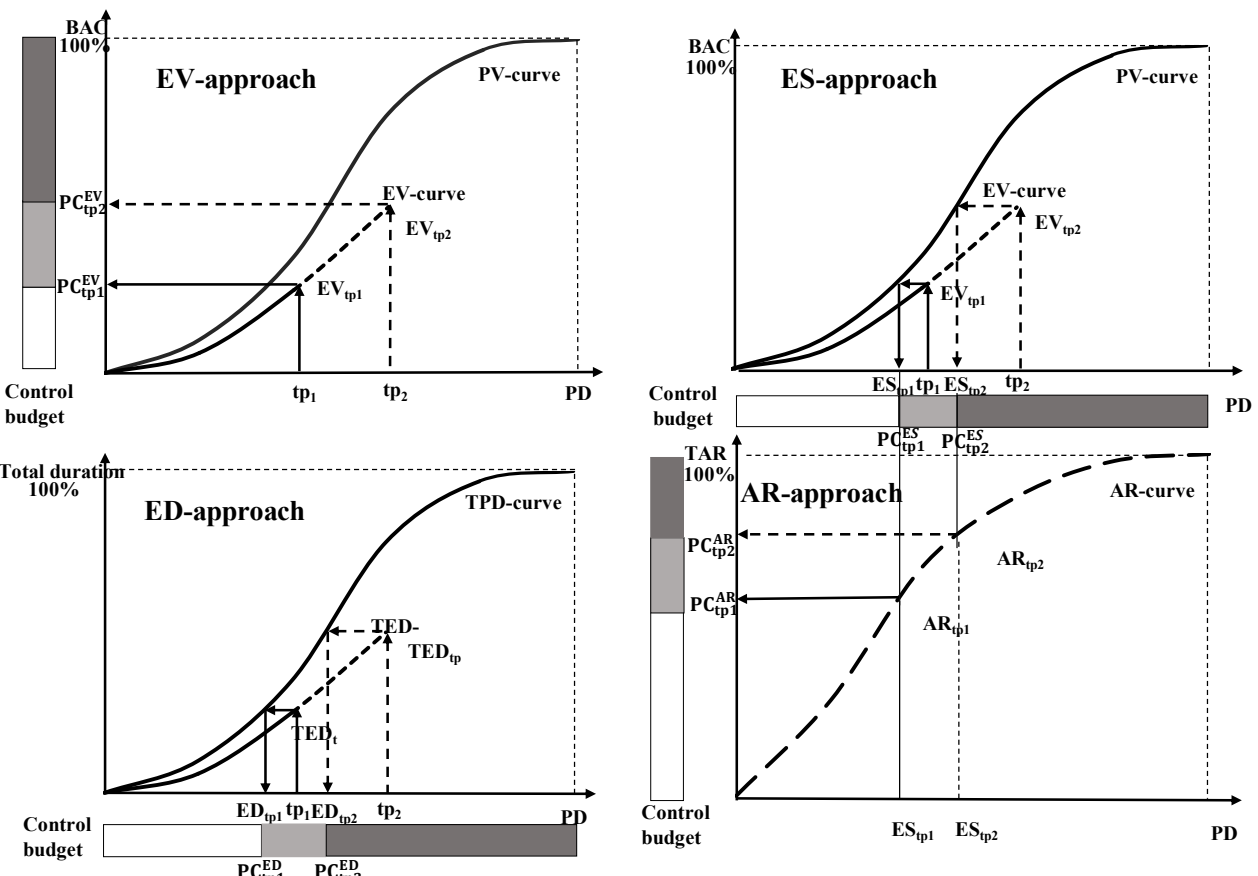

Figure 3: Determination of $A B_{t p}$

Earned value approach: For the earned value (EV) approach, the PC is calculated in a traditional way using the cost information of the project, which is measured as the EV/BAC with $\mathrm{EV}$ the earned value at the current time point and BAC the budget at completion, which is readily available in the scheduling phase. In order to distinguish the PC in different approaches, the $\mathrm{PC}$ of this $\mathrm{EV}$ approach is referred to as $P C^{E V}$ in the remainder of this paper. Accordingly, the control budget is assigned proportional to the $P C^{E V}$ of the project. More specifically, the amount of $A B_{t p}$ of this EV approach $\left(A B_{t p}^{E V}\right)$ is made available based on the expected $P C^{E V}$ of the next tracking period. The $A B_{t p}^{E V}$ is calculated as Eq. (2).

$$
A B_{t p}^{E V}=\left(P C_{t p+1}^{E V}-P C_{t p}^{E V}\right) \times \text { control budget }
$$


With $P C_{t p}^{E V}=\frac{E V_{t p}}{B A C}$.

The top left pane of Fig. 3 illustrates the determination of $A B_{t p 1}^{E V}$ graphically. For a fictitious project in progress, the current time is $t p_{1}$. In order to calculate the allowable budget consumption, $P C_{t p 1}^{E V}$ and $P C_{t p 2}^{E V}$ should be determined (Eq. (2)). Since $E V_{t p 1}$ is known at $t p_{1}, P C_{t p 1}^{E V}$ can be readily determined as $\frac{E V_{t p 1}}{B A C}$. However, for the next tracking period $\left(t p_{2}\right)$, the $\mathrm{EV}$ is not known yet. Therefore, the estimated $\mathrm{EV}$ at $t p_{2}\left(E V_{t p 2}\right)$ is used to determine $P C_{t p 2}^{E V}$. The resulting $A B_{t p 1}^{E V}\left(=\left(P C_{t p 2}^{E V}-P C_{t p 1}^{E V}\right) \times\right.$ control budget $)$ is depicted as a light grey box in Fig. 3.

Earned schedule approach: For the earned schedule (ES) approach, we propose a new PC measure taking the time/cost information of the project into account, the $P C^{E S}$, which is calculated as the ES/PD with ES the earned schedule at the current time point and PD the planned duration of the project at completion. Consequently, the control budget is allocated proportional to the $P C^{E S}$ of the project. More specifically, the amount of $A B_{t p}$ of this ES approach $\left(A B_{t p}^{E S}\right)$ that is made available is determined based on the expected $P C^{E S}$ of the next period. The $A B_{t p}^{E S}$ is calculated as shown in Eq. (3).

$$
A B_{t p}^{E S}=\left(P C_{t p+1}^{E S}-P C_{t p}^{E S}\right) \times \text { control budget }
$$

With $P C_{t p}^{E S}=\frac{E S_{t p}}{P D}$.

The top right pane of Fig. 3 illustrates the determination of $A B_{t p 1}^{E S}$ graphically. In order to calculate the allowable budget consumption, $P C_{t p 1}^{E S}$ and $P C_{t p 2}^{E S}$ should be determined (Eq. (3)). Since $E S_{t p 1}$ is known at $t p_{1}, P C_{t p 1}^{E S}$ can be readily determined as $\frac{E S_{t p 1}}{P D}$. However, for the next tracking period $\left(t p_{2}\right)$, the ES is not known yet. Therefore, the estimated ES at $t p_{2}$ $\left(E S_{t p 2}\right)$ is used to determine $P C_{t p 2}^{E S}$. The resulting $A B_{t p 1}^{E S}\left(=\left(P C_{t p 2}^{E S}-P C_{t p 1}^{E S}\right) \times\right.$ control budget $)$ is depicted as a light grey box in Fig. 3.

Earned duration approach: For the earned duration (ED) approach, we define the $P C^{E D}$ to measure the percentage completion of the project considering the time information of the project, which is calculated as the $\mathrm{ED} / \mathrm{PD}$ with $\mathrm{ED}$ the earned duration at the current time point. Thus the control budget is assigned proportional to the $P C^{E D}$ of the project. More specifically, the amount of $A B_{t p}$ of this ED approach $\left(A B_{t p}^{E D}\right)$ that is made available is determined based on the expected $P C^{E D}$ of the next period. The $A B_{t p}^{E D}$ is defined as follows:

$$
A B_{t p}^{E D}=\left(P C_{t p+1}^{E D}-P C_{t p}^{E D}\right) \times \text { control budget }
$$

With $P C_{t p}^{E D}=\frac{E D_{t p}}{P D}$.

The bottom left pane of Fig. 3 illustrates the determination of $A B_{t p 1}^{E D}$ graphically. To calculate the allowable budget consumption, $P C_{t p 1}^{E D}$ and $P C_{t p 2}^{E D}$ should be determined (Eq. (4)). Since 
$E D_{t p 1}$ is known at $t p_{1}, P C_{t p 1}^{E D}$ can be readily determined as $\frac{E D_{t p 1}}{T P D}$. However, for the next tracking period $\left(t p_{2}\right)$, the ED is not known yet. Therefore, the estimated ED at $t p_{2}\left(E D_{t p 2}\right)$ is used to define $P C_{t p 2}^{E D}$. The resulting $A B_{t p 1}^{E D}\left(=\left(P C_{t p 2}^{E D}-P C_{t p 1}^{E D}\right) \times\right.$ control budget $)$ is depicted as a light grey box in Fig. 3 .

Activity risk approach: For the Activity risk (AR) approach, we define the $P C^{A R}$ to measure the percentage completion of the project considering the activity risk information as well as the time/cost information of the project, which is calculated as the $A R_{t p} / \mathrm{TAR}$ with $A R_{t p}$ the total activity risk value of the project at the current time point and TAR the total activity risk value of the project at completion. Consequently, the control budget is assigned proportional to the $P C^{A R}$ of the project. More specifically, the amount of $A B_{t p}$ of this AR approach $\left(A B_{t p}^{A R}\right)$ that is made available is determined based on the expected $P C^{A R}$ of the next period. The $A B_{t p}^{A R}$ is calculated as Eq. (5).

$$
A B_{t p}^{A R}=\left(P C_{t p+1}^{A R}-P C_{t p}^{A R}\right) \times \text { control budget }
$$

With $P C_{t p}^{A R}=\frac{A R_{t p}}{T A R}$. Since the main goal of this paper is to allocate the control budget for top-down project control, the TAR is determined from the aggregated project level. In order to determine the TAR of the project, three steps are required, and the formulas used for each step are displayed in Table 3.

Table 3: Formulas for determining TAR of the project.

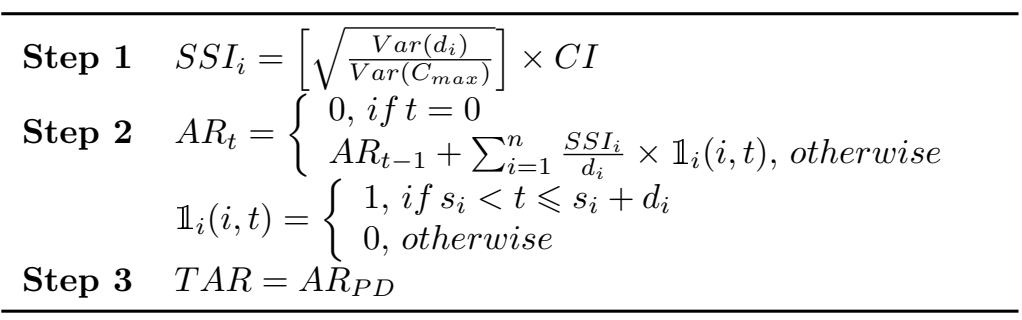

In the first step, the risk value of individual activity is calculated from Monte Carlo simulations before the project is executed. We use the Schedule Sensitivity Index (SSI) (PMBOK, 2004) to measure and evaluate the sensitivity of individual activity, since it measures the standard deviation of activity duration and the project duration $\left(S_{d i}\right.$ and $\left.S_{c \max }\right)$ with the criticality index $(C I)$. In the second step, we define the unit $S S I$ as the schedule sensitivity index of individual activity at each time unit, which is measured as $\frac{S S I_{i}}{d_{i}}$ (with $d_{i}$ the planned duration of each activity). Based on the unit SSI calculated from the first step, the $A R_{t}$ measures the cumulative risk of the activities in progress or finished at time t. Finally, the TAR of the project is calculated as the cumulative risk of the activities at time PD.

The determination of the $A B_{t p}$ is illustrated graphically in the bottom right pane of Fig. 3. It should be noted that the SSI value of each activity is obtained from Monte Carlo simulations 
before the start of the project, this is known as schedule risk analysis. The risk information of the project progress can be retrieved by means of the PV-curve, EV-curve and the AR-curve in an easy way. At $t p_{1}$, as $P C_{t p 1}^{E V}$ of the project is completed, which is planned to be completed at $E S_{t p 1}$, therefore, the $A R_{t p 1}$ can be found on the original AR-curve at $E S_{t p 1}$, which can be used to calculate the $P C_{t p 1}^{A R}\left(=\frac{A R_{E S t p 1}}{T A R}\right)$ of the TAR. In order to calculate the expected $P C_{t p 2}^{A R}$, the estimated $A R_{t p 2}$ is measured. Therefore, the $P C_{t p 2}^{A R}\left(=\frac{A R_{E S t p 2}}{T A R}\right)$. Accordingly, the $A R_{t p 1}$ is equal to the difference between $P C_{t p 1}^{A R}$ and $P C_{t p 2}^{A R}$ multiplying by the control budget.

\subsubsection{Three versions}

In the previous section, the control budget is allocated proportional to the PC of the project calculated in different ways along the whole project life cycle. However, allocating the control budget in a dynamically increasing or decreasing version according to the $\mathrm{PC}$ of the project might lead to more efficient actions and improve the project outcome. Consequently, in our computational experiments, these three alternative versions have been used. The formula of $A B_{t p}(\%)$ of the four approaches in three different versions are provided in Table 4. First, the so-called normal version, which has been described explicitly in Section 3.1.1, simply calculates the $\mathrm{AB}$ according to the formulas presented in the previous section. Second, an increasing version starts with a relatively low control budget allocation, but systematically increases the budget along the project progress. This increasing method is designed for the projects where the project information at the early stage is not adequate or sufficient to the project manager, but the quality improves along the project progress. Consequently, we will use the squared function with the $\mathrm{PC}$ as the base to make sure the control budget allocation at each project phase presents an increasing way and the total amount of budget allocation at the closure of the project is equal to $100 \%$. Finally, a decreasing version follows an opposite behavior, starting with a relatively high control budget allocation to solve problems early, but then systematically decreasing the available budget. This version is designed for projects when corrective actions at the early stage might have a significant impact on the rest of the project. Accordingly, we will employ the square root function of the PC, such that the budget allocation of each control phase presents a decreasing way and the total amount of budget allocation at the closure of the project is equal to $100 \%$.

\subsubsection{Illustrative example}

In this section, we use an illustrative example to demonstrate how the four approaches and three versions are applied to determine the allowable budget consumption during project execution. The project network, the baseline schedule, the SSI value of each activity, the PVcurve depicted according to the network, the TPD-curve delineated relying on the baseline 
Table 4: Formula of four methods in three versions.

\begin{tabular}{ccccc}
\hline Methods & PC & Normal & Versions & Increasing \\
\hline$E V$ & $P C_{t p}^{E V}=\frac{E V_{t p}}{B A C}$ & $P C_{t p+1}^{E V}-P C_{t p}^{E V}$ & $\left(P C_{t p+1}^{E V}\right)^{2}-\left(P C_{t p}^{E V}\right)^{2}$ & $\sqrt{P C_{t p+1}^{E V}}-\sqrt{P C_{t p}^{E V}}$ \\
$E S$ & $P C_{t p}^{E S}=\frac{E S_{t p}}{P D}$ & $P C_{t p+1}^{E S}-P C_{t p}^{E S}$ & $\left(P C_{t p+1}^{E S}\right)^{2}-\left(P C_{t p}^{E S}\right)^{2}$ & $\sqrt{P C_{t p+1}^{E S}}-\sqrt{P C_{t p}^{E S}}$ \\
$E D$ & $P C_{t p}^{E D}=\frac{E D_{t p}}{P D}$ & $P C_{t p+1}^{E D}-P C_{t p}^{E D}$ & $\left(P C_{t p+1}^{E D}\right)^{2}-\left(P C_{t p}^{E D}\right)^{2}$ & $\sqrt{P C_{t p+1}^{E D}}-\sqrt{P C_{t p}^{E D}}$ \\
$A R$ & $P C_{t p}^{A R}=\frac{A R_{t}}{T A R}$ & $P C_{t p+1}^{A R}-P C_{t p}^{A R}$ & $\left(P C_{t p+1}^{A R}\right)^{2}-\left(P C_{t p}^{A R}\right)^{2}$ & $\sqrt{P C_{t p+1}^{A R}}-\sqrt{P C_{t p}^{A R}}$ \\
\hline
\end{tabular}

schedule and the AR-curve determined from the SSI value of each activity are provided in Fig. 4. We assume the SSI value of individual activity is equal to $0.1,0.6,0.02$ and 0.2 respectively, but these values should of course be obtained by defining distributions on the activity durations and by using Monte Carlo simulations.

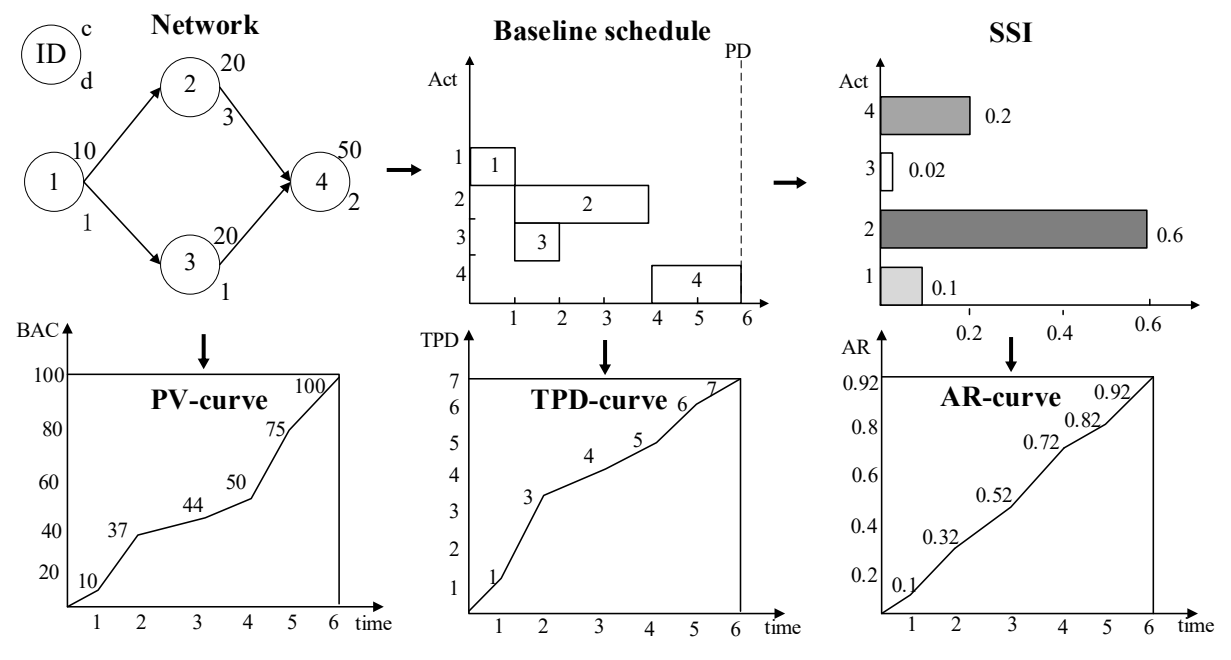

Figure 4: Before execution.

The fictitious project execution is presented on the left pane of Fig. 5. As shown in this figure, activity 1 has a delay of $50 \%$, the delay of activity 2 is $25 \%$ and activity 3 has a delay of $50 \%$. The progress of this fictitious project execution will be monitored and evaluated to determine the $A B_{t p}(\%)$ of the project. First, the PC calculated in different aspects at the current tracking period is measured according to the project progress. Subsequently, the expected $\mathrm{PC}$ of the next tracking period is determined. Finally, the $A B$ is set based on the calculated PC of these two tracking periods. The milestone tracking approach is employed in this example project. The first milestone of the project is set when activity 3 is completed, and the second milestone is set when activity 2 is completed, as shown on the right pane of Fig. 5 . 

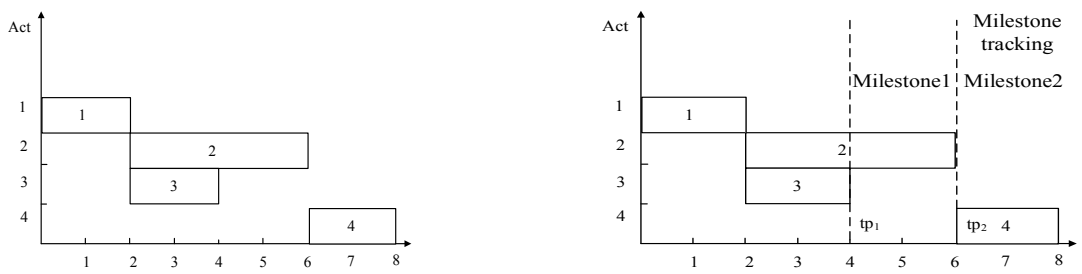

Figure 5: Project execution.

Milestone tracking: The left pane of Table 5 displays the monitored progress of the execution at each tracking period. The BAC, PD and TAR of the project which are readily available during the scheduling phase equal to 100, 6 and 0.92 respectively. Next, the specific calculation of the PC at each tracking period is demonstrated in detail.

- EV approach: The EV at $t p_{1}(\mathrm{t}=4)$ is equal to $40\left(=\operatorname{cost}_{\text {act } 1}+\operatorname{cost}_{\text {act } 2} \times \frac{2}{4}+\operatorname{cost}_{\text {act } 3}=\right.$ $\left.10+20 \times \frac{2}{4}+20\right)$, thus the $P C_{t p 1}^{E V}$ is equal to $40 \%\left(=\frac{E V}{B A C}=\frac{40}{100}\right)$. Similarly, the $P C_{t p 2}^{E V}$ is equal to $50 \%$.

- ES approach: The ES at $t p_{1}$ of the project is equal to $2.43\left(=t_{2}+\frac{E V-P V_{2}}{P V_{3}-P V_{2}}=2+\frac{40-37}{44-37}\right)$, hence, the $P C_{t p 1}^{E S}$ is equal to $41 \%\left(=\frac{E S}{P D}=\frac{2.43}{6}\right)$. Similarly, the $P C_{t p 2}^{E S}$ is equal to $67 \%$.

- ED approach: At $t p_{1}$, the TED of the project is equal to 3.5 (= duration act $1+$ duration $_{\text {act } 2} \times \frac{2}{4}+$ duration $_{\text {act } 3}=1+3 \times \frac{2}{4}+1$ ). Further, the ED of the project at $t p_{1}$ is equal to $2.5\left(=t_{2}+\frac{T E D-T P D_{2}}{T P D_{3}-T P D_{2}}=2+\frac{3.5-3}{4-3}\right)$, therefore the $P C_{t p 1}^{E D}$ is equal to $42 \%$ $\left(=\frac{E D}{P D}=\frac{2.5}{6}\right)$. Similarly, the $P C_{t p 2}^{E D}$ is equal to $67 \%$.

- AR approach: The AR of the project at $t p_{1}$ is determined at $E S_{t p 1}$. The $E S_{t p 1}$ has been determined from the ES approach, which is equal to 2.43. Thus, the AR at $t_{2.43}$ is equal to $0.41\left(=0.1+0.6 \times \frac{2.43-1}{3}+0.02\right)$. The $P C_{t p 1}^{A R}$ is equal to $45 \%\left(=\frac{0.41}{0.92}\right)$. Similarly, the $P C_{t p 2}^{A R}$ is equal to $89 \%$.

Table 5: Progress metrics and $A B_{t p 1}$ in three versions.

\begin{tabular}{|c|c|c|c|c|c|c|c|}
\hline & \multicolumn{4}{|c|}{ Progress metrics } & \multicolumn{3}{|c|}{$A B_{t p 1}(\%)$} \\
\hline & \multicolumn{2}{|c|}{ Values } & \multicolumn{2}{|c|}{$\mathrm{PC}(\%)$} & \multicolumn{3}{|c|}{ three versions } \\
\hline & $t p_{1}$ & $t p_{2}$ & $t p_{1}$ & $t p_{2}$ & Normal & Increasing & Decreasing \\
\hline $\mathrm{EV}$ & 40 & 50 & 40 & 50 & 10 & 9 & 50 \\
\hline $\mathrm{ES}$ & 2.43 & 4 & 41 & 67 & 26 & 28 & 18 \\
\hline $\mathrm{ED}$ & 2.50 & 4 & 42 & 67 & 25 & 27 & 17 \\
\hline $\mathrm{AR}$ & 0.41 & 0.82 & 45 & 89 & 44 & 59 & 27 \\
\hline
\end{tabular}

The $A B_{t p 1}$ of each approach for the three versions is listed on the right pane of Table 5 . The $A B_{t p 1}$ in the normal version is calculated as the difference between the $\mathrm{PC}$ of the first two tracking periods. For instance, the allowable budget consumption at $t p_{1}$ is $10 \%(=50 \%-40 \%)$ 
for the EV approach, $26 \%(=67 \%-41 \%)$ for the ES approach, $25 \%(=67 \%-42 \%), 44 \%$ $(=89 \%-45 \%)$ for the AR approach. For the increasing and decreasing versions, the formulas of Table 2 are used.

\subsection{Corrective action}

When the control budget is allocated to each control phase and the measured project performance is lower than the corresponding tolerance limits, the project manager has to decide which activities are eligible to take corrective actions on and then verify whether the allowable budget consumption is adequate or not. Consequently, two steps are required during this action phase.

In the first step, the eligible activities at each tracking period (tp) should be determined when an action is needed. We will focus on the critical activities in progress at that tracking period in order to reduce the estimated remaining duration of these activities to further improve the project outcome.

In the second step, the allowable budget consumption should be compared with the total cost of crashing $(T C)$, which stipulates the necessary cost to take corrective actions on the eligible activities. The total cost of crashing is affected by the reduction in activity duration and the extra cost of the reduction. First, the reduction in activity duration (Reduction) is restricted to a fixed percentage of the estimated remaining duration of the eligible activity. Subsequently, the extra cost (Extra cost) is defined as the additional cost to decrease the activity duration for each time unit. This additional cost is incurred since the project manager needs to exploit extra resources (reassignment of highly-skilled personnel, extra equipments, improvement of manpower, etc.) to crash eligible activities, which is always associated with an increased cost. Finally, the total cost of crashing is calculated by multiplying the reduction in activity duration with the extra cost (Eq. (6)). When an action is needed and the total cost of crashing of that tracking period is within the allowable budget consumption, the activity duration will be reduced under the strict predefined limits.

$$
T C_{t p}=\text { Reduction }_{t p} \times{\text { Extra } \text { cost }_{t p}}
$$

\subsection{Simulation model}

With the aim of generating a large set of fictitious project executions, Monte Carlo simulations are employed to generate project information with the presence of uncertainty. We will use the lognormal distribution which is skewed to the right to model the real activity duration (Bie et al., 2012; Hu et al., 2016; Kotiah and Wallace, 1973; Martens and Vanhoucke, 2018), with $\mu=1.1$ and $\delta=0.3$. The indicators and parameters used in the simulation model are 
summarized in Table 6, the parameters are varied and controlled for the four approaches in three versions (Section 3.1).

Table 6: Specific settings for the parameters.

\begin{tabular}{lll}
\hline Indicators & Classification & Values \\
Project network & SP & $\{0.1,0.2, \ldots, 0.9\}$ \\
Activity & Number & 30 \\
& Cost & {$[100,900]$} \\
& Resource & $/$ \\
\hline Parameters & & Values \\
\hline Control budget & & $10 \%, 12 \%, 14 \%$ \\
Tolerance limits & $15 \%, 25 \%, 35 \%$ \\
Corrective actions & & $30 \%, 50 \%, 70 \%$ \\
\hline
\end{tabular}

Indicators: In order to test the impact of control budget on the corrective actions for a wide variety of projects, a set of 900 fictitious project networks with topological structure are generated by a project network generator RanGen (Demeulemeester et al., 2003; Vanhoucke et al., 2008). The dataset is extensively applied in previous project control simulation studies (Ballesteros-Pérez et al., 2019; Elshaer, 2013). The topological structure of these fictitious project networks are presented by the serial/parallel (SP) indicator, which is used to measure the closeness of a project network, and contains 100 projects for $\mathrm{SP}=\{0.1,0.2, \ldots, 0.9\}$. More specifically, project networks with low (high) SP values are close to parallel (serial) projects. Each project network consists of 30 activities. For each activity, the fixed cost is uniformly sampled between $€ 10$ and $€ 90$, a variable cost is uniformly sampled between $€ 100$ and $€ 900$.

Parameters: The parameters are used to model budget allocation and corrective action taking process. In order to investigate the impact of an increasing control budget on the expected project outcome, we model the control budget of $10 \%, 12 \%$, and $14 \%$ of BAC to perform possible corrective actions. During project execution, we construct tolerance limits by assuming that the project buffer of a certain percentage of the planned duration is consumed proportionally to the PV accrue of each project phase. More specifically, when the project is completed at $\mathrm{x} \%$ of the BAC, $\mathrm{x} \%$ of the project buffer is allowed to be consumed for that phase. This approach is developed by Martens and Vanhoucke (2017a). These so-called ES-based tolerance limits are used in the EV, ES and AR approaches to monitor the project progress. While the ED approach employs the ED-based tolerance limits, which simply changes the PV accrue used in the ES-based tolerance limits into the PD accrue. In the simulation model, the tolerance limits are set for project buffer sizes of $15 \%, 25 \%$ and $35 \%$ of the PD to simulate the frequency of warning signals in the project. When a warning signal is generated, an action will be taken only if the allowable budget consumption is adequate at that control phase. In this case, the estimated remaining duration of the eligible activity will be reduced under 
strict predefined limits. In the simulation experiments, three reduced levels of duration are considered and compared (i.e. $30 \%, 50 \%$ and $70 \%$ of the estimated activity duration) in order to simulate different degrees of corrective actions.

\subsection{Performance measurement}

In order to measure and compare the impact of the four control budget allocation approaches on the corrective action taking process, two types of performance metrics are proposed, namely the efficiency and the effectiveness. In an economic context, the efficiency is entirely different from the effectiveness. The efficiency is defined as operating in the best possible way with the least waste of effort, by measuring the ratio between the total output and the total input. The effectiveness is defined as accomplishing the expected results, by measuring the extents of the expected results that have been achieved. In order to illustrate the difference between the efficiency and the effectiveness, the left-right direction in Fig. 6 represents the degree of efficiency, from inefficient to efficient. The bottom-up direction denotes the degree of the effectiveness, from ineffective to effective. Four combined results are provided in the box in detail.

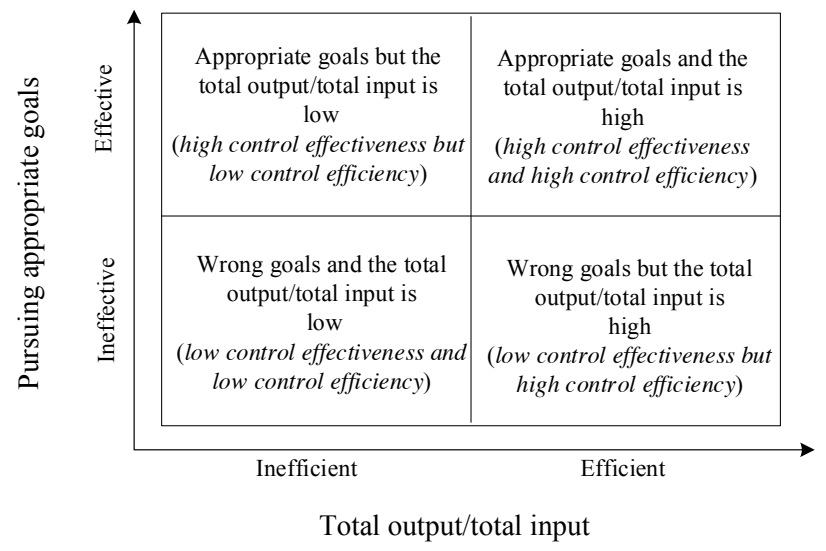

Figure 6: The difference between (control) efficiency and (control) effectiveness.

In the context of project control, the control efficiency is used to evaluate the ability of each approach to reduce the real project duration (total output) within the same control budget (total input). The control effectiveness measures the extent of project delay (appropriate goals) that have been reduced by taking corrective actions. In Fig. 6, four combined situations are presented, namely, high control effectiveness but low control efficiency, high control effectiveness and high control efficiency, low control effectiveness and low control efficiency, low control effectiveness but high control efficiency. While a high control efficiency is not necessarily in accordance with a high control effectiveness, it is of major importance to combine 
the control efficiency and the control effectiveness to pursue the appropriate goals with the least waste of effort. Therefore, the ultimate goal is to explore an approach which considers both the control efficiency and control effectiveness (the right above box in Fig. 6). An overview of abbreviations used in this section is provided in Table 7. The control efficiency and control effectiveness are discussed in greater detail in the remainder of this section.

Table 7: Abbreviations used for performance measurement

\begin{tabular}{ll}
\hline Abbreviations & Explanations \\
\hline$n$ ract & Number of activities in each project \\
$R D^{\text {no }}$ & Real duration of the project without corrective actions \\
$R D^{y e s}$ & Real duration of the project with corrective actions taken \\
$R D_{i}^{n o}$ & Actual duration of activity i without corrective actions \\
$R D_{i}^{\text {yes }}$ & Actual duration of activity i with corrective actions \\
$n r s_{w}$ & Number of projects with warning signals in the simulation \\
$n r s_{l}$ & Number of delayed projects in the simulation \\
Delayo & Project delay without corrective actions \\
Delay yes & Project delay with corrective actions taken \\
DL & Deadline of the project \\
\hline
\end{tabular}

- Control efficiency: The control efficiency indicates the average return of corrective actions by comparing the total reduction in project duration with the total control budget invested in the projects. Consequently, we take all projects that generate warning signals into account, even when these projects have no delay compared to the baseline schedule. Since corrective actions affect both the time and cost of a project, the control efficiency will be evaluated from both the time and cost aspect. These two aspects of control efficiency are described as follows:

Time efficiency: The time efficiency evaluates the control efficiency from the time perspective by measuring the total reduction in the project real duration after taking corrective actions $\left(R D^{n o}-R D^{y e s}\right)$ compared to the sum of reduction in the activities due to crashing $\left(R D_{i}^{n o}-R D_{i}^{y e s}\right)$, as described in Eq. (7).

$$
\text { time ef ficiency }=\left\{\begin{array}{l}
0, \text { if denominator }=0 \\
\frac{1}{n r s_{w}} \sum_{k=1}^{n r s_{w}}\left(\frac{R D^{n o}-R D^{y e s}}{\sum_{i=1}^{n r a c t}\left(R D_{i}^{n o}-R D_{i}^{\text {yes }}\right)}\right) \text {, otherwise }
\end{array}\right.
$$

With $n r s_{w}$ the total number of projects with warning signals. As can be seen from this formula, the time efficiency equals to 1 when the reduction in real project duration is equal to the total change in all evaluated activities, which is a desirable state. Moreover, the time efficiency can be also equal to 0 , when the total change in all the activities has no effect on the real project duration (numerator $=0$ ) or when no corrective actions are taken when warning signals generated, due to a lack of available control budget $($ denominator $=0)$.

Cost efficiency: The cost efficiency indicates the reduction of project duration per invested monetary unit by comparing the total reduction in real project duration after actions $\left(R D^{n o}-R D^{y e s}\right)$ to the total amount of cost incurred for corrective actions (total 
crash cost) (Eq. (8)).

$$
\text { costefficiency }=\left\{\begin{array}{l}
0, \text { if denominator }=0 \\
\frac{1}{n r s_{w}} \sum_{k=1}^{n r s_{w}}\left(\frac{R D^{n o}-R D^{\text {yes }}}{\text { total crash cost }}\right) \text {, otherwise }
\end{array}\right.
$$

Since the reduction in project duration is significantly lower than the total crash cost, the cost efficiency is much smaller than 1 . The cost efficiency can be also equal to 0 , when the total crash cost has no effect on the real project duration (numerator $=0$ ) and when no crash cost is spent, owing to a lack of available control budget (denominator $=0)$.

- Control effectiveness: The control effectiveness indicates the total reduction in project delay due to the corrective actions. Accordingly, we only consider the projects with the presence of delays. Further, the project delay can be measured at two distinguished levels. At the first level, we evaluate whether the project delay without corrective actions could be avoided by taking corrective actions. In other words, we will examine how much of the delayed projects could be completed on time by taking corrective actions. At the second level, the amount of delay reduction will be considered to evaluate the average amount of delay reduction. Therefore, the control effectiveness is measured at these two levels by means of the timely completion improvement and crashing effectiveness respectively.

Timely completion improvement: The timely completion improvement compares the number of delayed projects completed before the deadline (DL) thanks to the corrective actions $\left(\mathbb{1}_{i}\left(R D^{n o}>D L\right) \mathbb{1}_{i}\left(R D^{\text {yes }} \leqslant D L\right)\right.$, to the sum of the delayed projects before any action is made $\left(\mathbb{1}_{i}\left(R D^{n o}>D L\right)\right)$, without taking into account the precise reduction of project delay, as presented in Eq. (9).

$$
\text { Timely completion improvement }=\frac{\sum_{k=1}^{n r s_{l}} \mathbb{1}_{i}\left(R D^{n o}>D L\right) \mathbb{1}_{i}\left(R D^{\text {yes }} \leqslant D L\right)}{\sum_{k=1}^{n r s_{l}} \mathbb{1}_{i}\left(R D^{n o}>D L\right)}
$$

With $n r s_{l}$ the total number of late projects and $\mathbb{1}_{i}(\mathrm{~A})$ denotes the identity operator, which returns 1 if $\mathrm{A}$ is true, or else 0 . The timely completion improvement can be equal to 1 when all the delayed projects are completed timely after the corrective actions, which is a desirable state. It can be also equal to 0 , when no projects are completed before the expected due date after taking corrective actions.

Crashing effectiveness: The crashing effectiveness calculates the ratio between the reduction in project delay due to corrective actions $\left(\right.$ Delay $^{n o}-$ Delay $\left.^{y e s}\right)$ and the total 
delay before corrective actions $\left(\right.$ Delay $\left.^{n o}\right)$ along the project progress (Eq. (10)).

$$
\text { Crashingeffectiveness }=\frac{1}{n r s_{l}} \sum_{k=1}^{n r s_{l}}\left(\frac{\text { Delay }^{n o}-\text { Delay }^{\text {yes }}}{\text { Delay }^{n o}}\right)
$$

The crashing effectiveness can be equal to 1 when the project delay is repaired by the control budget entirely, which is a perfect state. It can also be equal to 0 , when no project delay is reduced after taking corrective actions.

\section{Results analysis}

In this section, various simulation results are discussed based on the different simulation runs and parameter settings discussed in Section 3.3, deploying the performance metrics proposed in Section 3.4.

Table 8: Specific settings for the parameters.

\begin{tabular}{|c|c|c|c|c|c|c|}
\hline & Approach & Version & SP & $\mathrm{BS}(\%)^{\mathrm{a}}$ & $\mathrm{CA}(\%)^{\mathrm{b}}$ & $\mathrm{CB}(\%)^{\mathrm{c}}$ \\
\hline Section 4.1 & 4 & 3 & $0.1-0.9$ & 25 & 50 & 12 \\
\hline Section 4.2 & ED & 3 & $0.1-0.9$ & 25 & 50 & 12 \\
\hline Section 4.3 & 4 & Increasing & $0.1-0.9$ & 25 & 50 & 12 \\
\hline Section 4.4 & 4 & Increasing & $0.1-0.9$ & $15,25,35$ & 50 & 12 \\
\hline Section 4.5 & 4 & Increasing & $0.1-0.9$ & 25 & $30,50,70$ & 12 \\
\hline Section 4.6 & 4 & Increasing & $0.1-0.9$ & 25 & 50 & $10,12,14$ \\
\hline
\end{tabular}

Table 8 summarizes the parameter settings for the experiments conducted in this section. The main focus of each section is highlighted in bold. First, the impact of different control budget allocation approaches on the project performance is reviewed in Section 4.1. Subsequently, a comparison of these four approaches used in three versions is investigated in Section 4.2. The effect of different project networks on the performance is the subject of Section 4.3. Section 4.4 examines the influence of different project buffer sizes on the project performance. Section 4.5 evaluates the impact of different levels of corrective actions on the project performance. The effect of an increased level of available control budget on the performance is investigated in Section 4.6. Finally, based on the results obtained from previous sections, a combined approach is developed in Section 4.7. Table 9 shows a summary of the results of our computational experiments that will be discussed in the next sections. Each time, the key results are briefly mentioned, and compared with the literature. Finally, a conclusion with advice is drawn for use in practice. 
Table 9: Summary

\begin{tabular}{|c|c|c|c|}
\hline Section & Key results & Comparison with the literature & Advice \\
\hline Section 4.1 & $\begin{array}{l}\text { The AR approach is better than the } \\
\text { ES approach on average. }\end{array}$ & $\begin{array}{l}\text { In line with the predictive capability of the DPI } \\
\text { (Khamooshi and Golafshani, 2014). } \\
\text { In line with Hu et al. (2016), where the corrective } \\
\text { actions with SRA perform better than the same ap- } \\
\text { proach without SRA. }\end{array}$ & $\begin{array}{l}\text { The ED approach performs well in project con- } \\
\text { trol. }\end{array}$ \\
\hline Section 4.2 & The increasing version performs best. & $\begin{array}{l}\text { Contrary to Vanhoucke (2011), where the decreasing } \\
\text { version is better for top-down control. }\end{array}$ & $\begin{array}{l}\text { The increasing version is preferred for top-down } \\
\text { control with limited budget, while the decreasing } \\
\text { version is recommended for the top-down control } \\
\text { with unlimited budget. }\end{array}$ \\
\hline Section 4.3 & $\begin{array}{l}\text { The efficiency is better for serial } \\
\text { projects. } \\
\text { The ED approach improves the effec- } \\
\text { tiveness in parallel projects. } \\
\text { The AR approach performs better in } \\
\text { serial projects, while the ES approach } \\
\text { is better for parallel projects. }\end{array}$ & $\begin{array}{l}\text { Contrary to Vanhoucke }(2011) \text {, where the bottom- } \\
\text { up control is better for parallel projects. } \\
\text { In line with Elshaer (2013), where the EVM-based } \\
\text { approach with SRA is better than that without SRA } \\
\text { in the early stage. }\end{array}$ & \\
\hline Section 4.4 & $\begin{array}{l}\text { The efficiency decreases when the } \\
\text { buffer size grows, while the effective- } \\
\text { ness presents an opposite behavior. }\end{array}$ & & \\
\hline Section 4.5 & $\begin{array}{l}\text { The performance is better for the cor- } \\
\text { rective actions with shorter reduction. }\end{array}$ & & $\begin{array}{l}\text { Frequent corrective actions with small reduction } \\
\text { are suggested for top-down control with limited } \\
\text { budget. }\end{array}$ \\
\hline Section 4.6 & $\begin{array}{l}\text { More control budget is better for the } \\
\text { performance. }\end{array}$ & & \\
\hline Section 4.7 & $\begin{array}{l}\text { The combined approach is better for } \\
\text { serial projects, the ED approach is } \\
\text { better for the parallel projects. }\end{array}$ & & $\begin{array}{l}\text { The combined approach is better for very serial } \\
\text { projects, while the ED approach is preferred for } \\
\text { parallel projects. }\end{array}$ \\
\hline
\end{tabular}



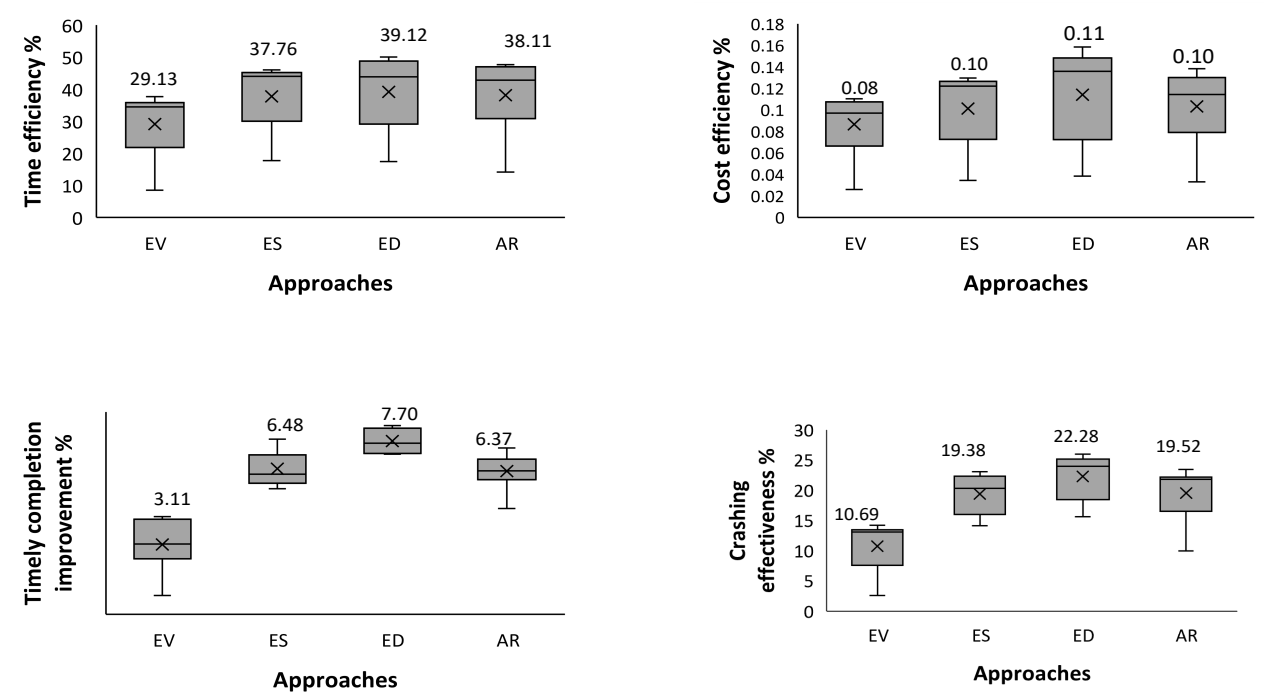

Figure 7: Comparison of different approaches.

\subsection{Comparison of different approaches}

Fig. 7 displays the results for EV, ES, ED and AR approaches implemented in three different versions (normal, increasing, decreasing) using the box plots with crosses representing the average performance. Results can be summarized along the following lines.

First, both the control efficiency (i.e. time efficiency 39.12, cost efficiency 0.11, Avg) and the control effectiveness (i.e. timely completion improvement 7.70, crashing effectiveness 22.28, Avg) are relatively higher for the ED approach, compared to the other three approaches. These results show that measuring the project progress using the ED-DPI metrics in combination with assessing this progress by means of the ED-based tolerance limits on average lead to more reliable indications of project problems during the project execution. This is in line with the results found in Batselier and Vanhoucke (2015), where the predictive capabilities of the ED-DPI have shown a slight advantage over the ES-SPI(t) using empirical data. Since the main goal of our current research is to study the impact of control budget allocation on the corrective action taking process, the results should be further reviewed by means of different ways of budget allocation. In case of the ED approach, more control budget will be allocated to project control phases with longer earned duration, which has a significant effect on the project duration. Consequently, more reduction in project delay due to more allowable budget consumption of that control phase is beneficial for the expected project outcome. However, in case of the EV approach, more control budget will be allocated to control phases with more earned value accrue, which might have a minor impact on the project deadline (i.e. project phases with activities with short duration but high cost of crashing). This is exactly 
the reason why the EV approach performs worst. Consequently, the ED approach is not only efficient in saving effort of the project manager but also effective in reducing the project delay, which is validated to be an alternative approach in the project control systems.

Second, Fig. 7 demonstrates that the performance of the AR approach (using the SSI information to allocate control budget, setting ES-based tolerance limits and measuring the project progress with the SPI(t)) presents a slight advantage over that of the ES approach (using the ES information to allocate control budget, setting ES-based tolerance limits and measuring the project progress with the $\mathrm{SPI}(\mathrm{t})$ ), which is consistent with $\mathrm{Hu}$ et al. (2016), who state that the results of corrective actions considering the SRA information perform slightly better on average than that of the same approach without taking the SRA information into account. The variability of the AR approach is much higher than that of the ES approach, which is affected by the project network structure and will be assessed in Section 4.3.

\subsection{Comparison of three versions}

Fig. 8 shows the allocated budget at each tracking period $\left(A B_{t p}\right)$ for the normal, increasing and decreasing version for the four methods of all the projects used in our experiments (average values are shown). This figure clearly shows that the three versions indeed approximately allocate the limited budget evenly across the whole project life cycle (normal version), or spread most of the limited budget in the late stages (increasing version) or early stages (decreasing version). Fig. 9 displays the results for these three versions for the ED method.

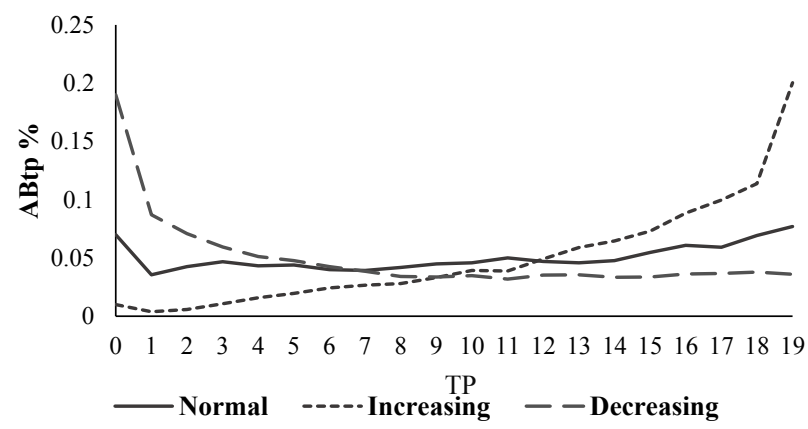

Figure 8: The general trend of allocating budget in three versions.

These graphs demonstrate that the ED approach performed in an increasing version (i.e. 7.70) outperforms the normal and decreasing versions (i.e. 4.97, 3.08) in terms of the control 

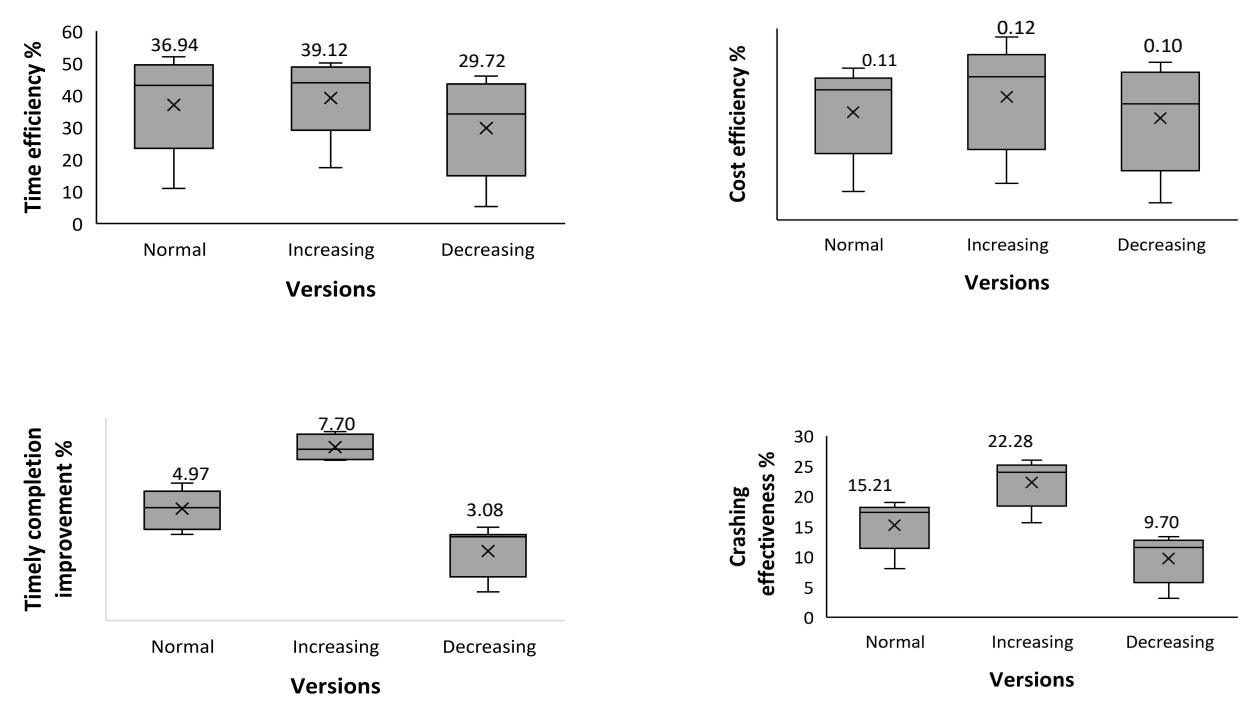

Figure 9: Comparison of different versions.

efficiency and control effectiveness. In case of the increasing version, we invest a lot of control budget to the late project stages rather than the initial stages in the project progress to revise the project delay. This is in contrast to the study of Vanhoucke (2011), who argues that gradually decreasing the attention of the project manager to the late stages is more beneficial for the outcome in top-down project control. This is due to the fact that the limited availability of control budget for solving problems is considered in the current paper, rather than employing unlimited control budget to take all possible corrective actions to evaluate the efficiency during the project execution. Accordingly, if all possible corrective actions can be taken in top-down project control, it is better to dynamically decrease the effort along the project progress. However, the availability of the control budget for actions is always a limitation for real-life projects, increasing the attention of the project manager along the project execution is thus preferred.

\subsection{Impact of project networks}

Fig. 10 displays the results of the four approaches for projects with different network topologies. The results can be summarized along the following lines. First, the control efficiency is much more beneficial for projects with more serial activities in general, since the EVM-based approaches perform traditionally well in serial projects (Vanhoucke and Vandevoorde, 2007). However, our study shows that the control effectiveness of the ED approach implemented in an increasing version significantly improves the control effectiveness in parallel projects rather than the serial ones compared with the other approaches. Second, the control efficiency and effectiveness of the AR approach change remarkably when SP values grow. More specifically, 

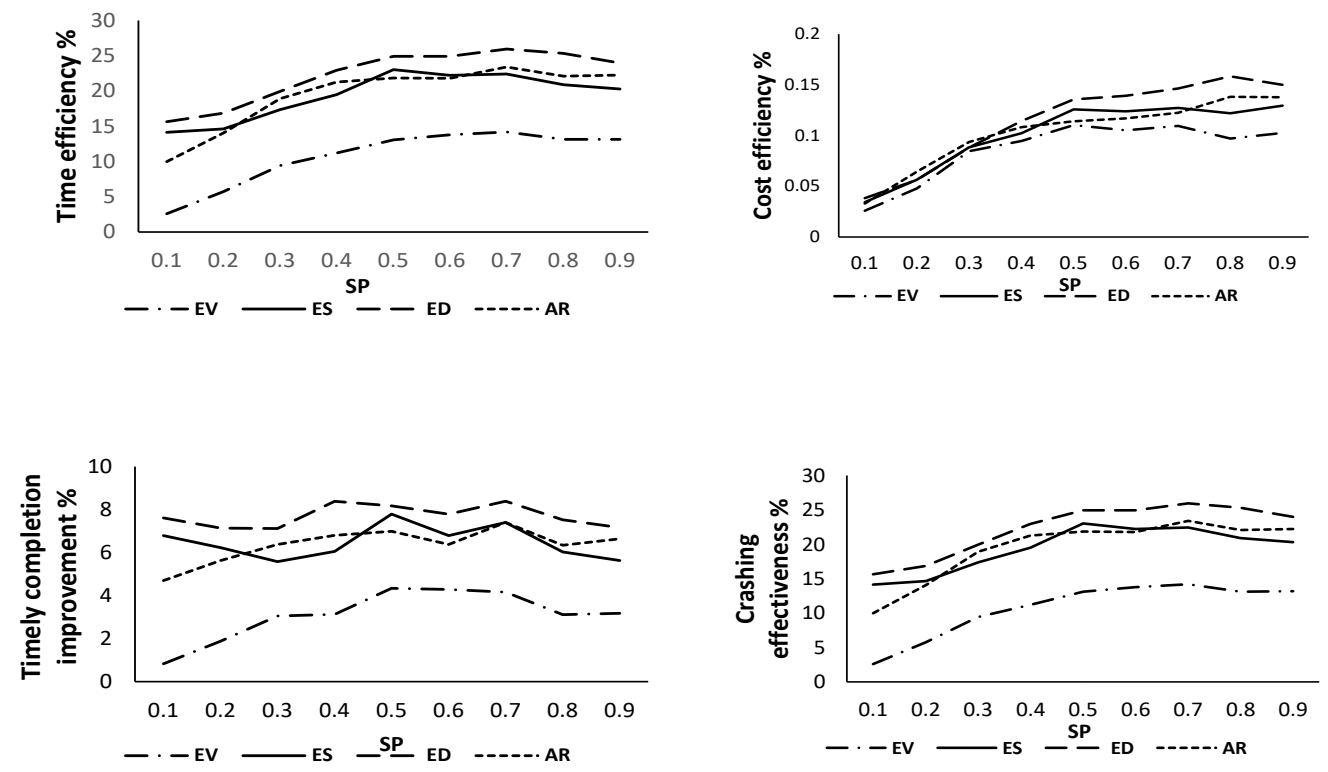

Figure 10: Impact of project networks on performance.

the AR approach is more beneficial for serial projects. This observation is in contrast to the conclusion obtained from Vanhoucke (2011) where it has been presented that the bottom-up tracking approach using the SRA information is particularly efficient for parallel projects. The main reason for this difference is that our AR approach is used for top-down project control rather than bottom-up control and it is shown that the top-down control performs better for serial projects. From this perspective, our results are not in contrast but in line with results from literature.

Finally, the performance of the AR approach (i.e. crashing effectiveness, 9.97) is relatively lower than that of the ES approach (i.e. 14.14) in parallel projects, while the results of the AR approach (i.e. 22.25) present a slight advantage over that of the ES approach (i.e. 20.30) for serial projects. In order to save the computational time, we did not dynamically update the activity $S S I$ values at each tracking period. However, an interesting behavior is found here. When project activities are executed in parallel, the critical path is frequently changed after taking corrective actions, which has a significant effect on the SSI values of certain activities. In these cases, the necessity to resimulate the processing and future activities to update the corresponding $S S I$ values increases. However, when project activities are executed in series, the critical path is seldom changed after taking corrective actions, which has little or no impact on the $S S I$ values of each activity. In these cases, the necessity to resimulate the processing and future activities to update the corresponding $S S I$ values is low. Hence, allocating the control budget in proportional with activity's risk accrue often lead to 

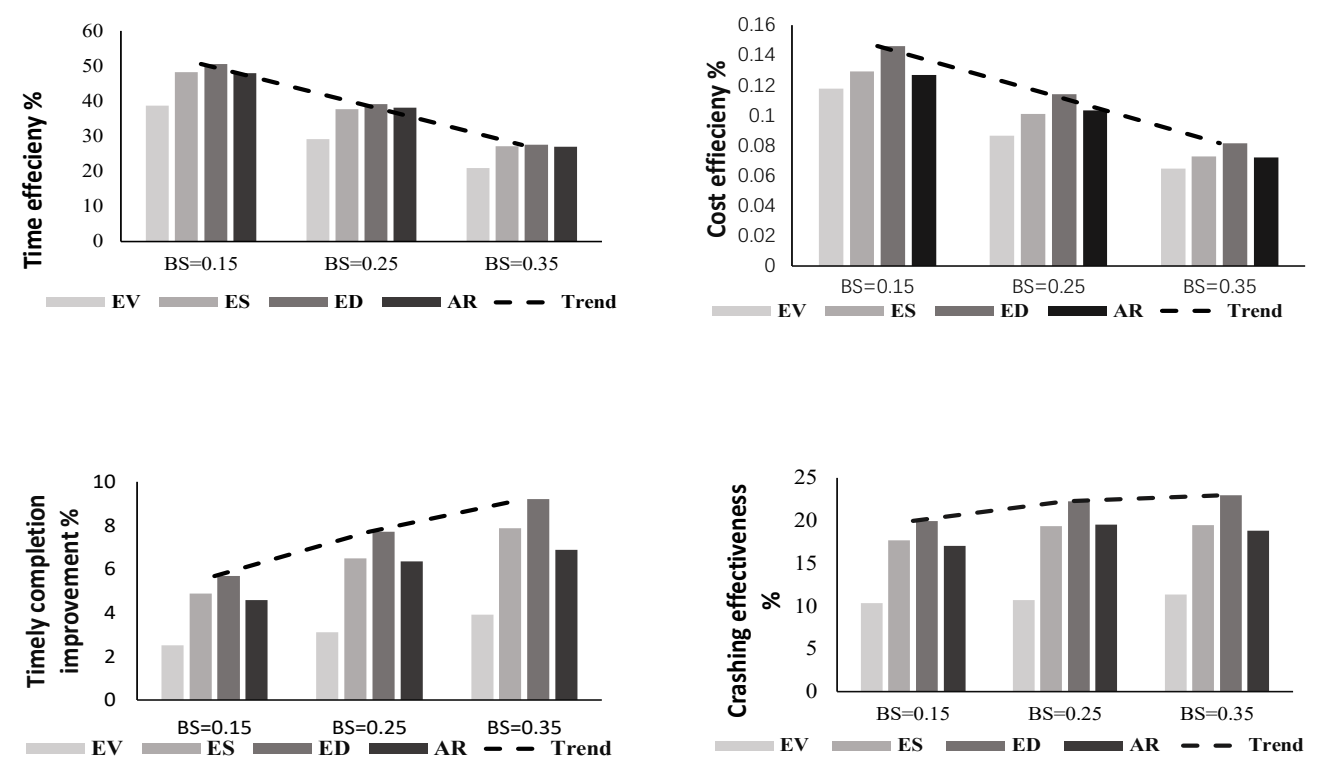

Figure 11: Impact of buffer sizes on performance.

an increased control efficiency and effectiveness in serial projects. This is exactly the reason why the AR approach performs relatively better than the ES approach on average (discussed in Section 4.1). Moreover, our conslusion is consistent with the behavior found in Batselier and Vanhoucke (2015), where the ES forecasting approach integrating the SRA information (Elshaer, 2013) is much more beneficial than the predictive capabilities of the ES-SPI(t) and ED-DPI, mostly due to the good performance at the early stage.

\subsection{Impact of buffer sizes}

Fig. 11 displays the results for different values of buffer sizes. The control efficiency decreases when the buffer size grows. When a relatively small buffer size is added at the end of the project, the SPI $(\mathrm{t})$ or DPI measures will be frequently exceeded by the dynamic tolerance limits, and even the presence of small delays in the projects will result in more corrective actions in dynamic project progress. However, in case of a relatively large buffer size, the project performance measures seldom drop below the tolerance limits to generate warning signals to take corrective actions. In these cases, a false signal (i.e. project delay due to serious delay in non-critical activities which has little impact on the project duration) will have a larger influence (negative) on the control efficiency of projects with large buffer sizes than the projects with small buffer sizes. While the graphs illustrate that the control effectiveness presents an opposite behavior compared with the control efficiency. In these cases, projects with small buffer sizes exhibit more serious delays than the projects with large buffer sizes 

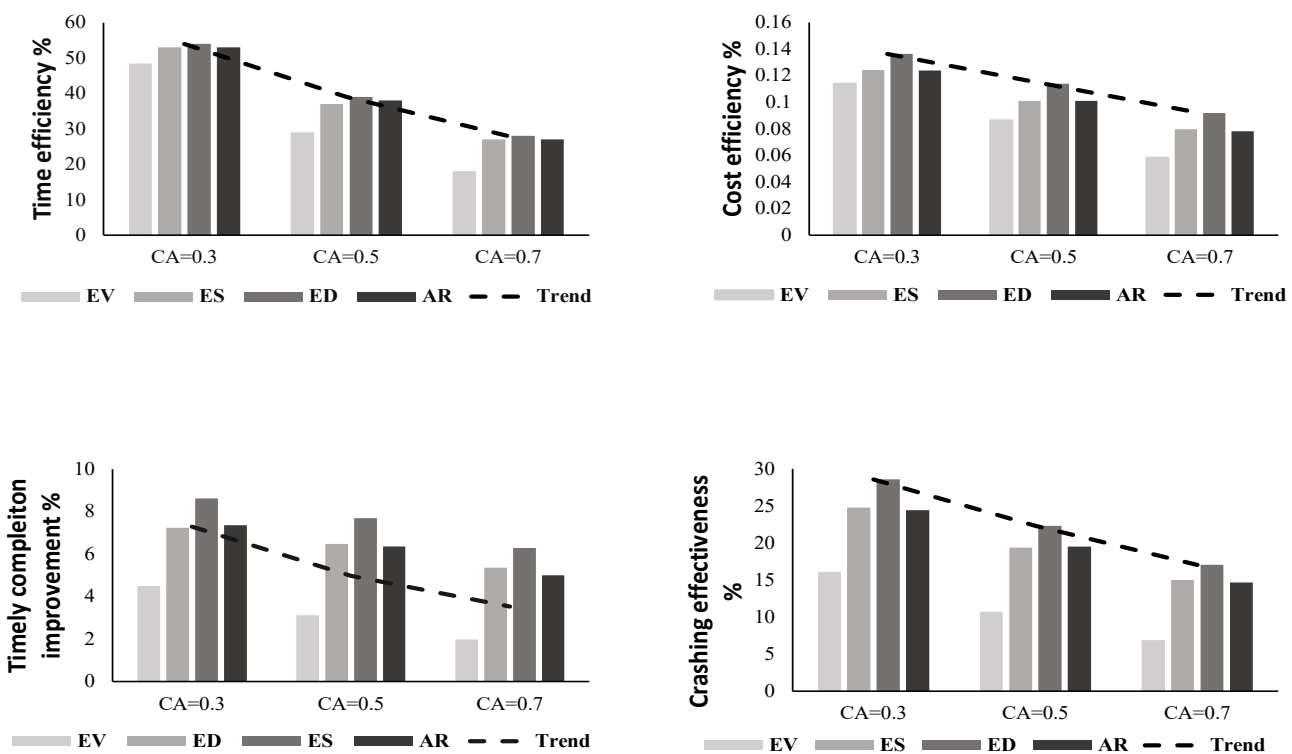

Figure 12: Impact of corrective actions on performance.

before taking corrective actions. Even the small buffer sizes always lead to more corrective actions during the project progress as described previously, this impact is not as substantial or significant as the impact of the project delay due to different buffer sizes on the control effectiveness.

Overall, in situations where the control budget (resources) is abundant to take corrective actions and project delays are unacceptable, we are inclined to recommend the use of tolerance limits with larger buffer sizes in order to meet the expected project deadline. However, the control budget (resources) is always limited during the project execution in practice. It is of major importance to use the tolerance limits with relatively small buffer sizes, to make the best use of the limited resources.

\subsection{Impact of corrective actions}

Fig. 12 displays the results for different amounts of corrective actions. The control efficiency and effectiveness is particularly beneficial for projects with relatively small reductions. A relatively small reduction in activity duration always corresponds to less total cost of crashing, which requires less allowable budget consumption at that control phase as well. This often results in the delayed project being repaired frequently with small reductions, which has a significant impact on the project duration. Contrarily, a relatively significant reduction in activity duration is always in accordance with more total cost of crashing, which requires 
more allowable budget consumption at that project phase. The project delay is seldom being revised with significant reduction, due to the limited budget, which has a minor impact on the project duration. Consequently, it is better to take corrective actions with small reduction frequently, rather than seldom performing actions with large reduction with the restriction of control budget.

\subsection{Impact of control budget}

Fig. 13 explicitly shows the results for different amounts of control budget. The control efficiency and effectiveness presents an increasing trend with more control budget invested. This observation coincides with the common sense, the more control budget invested during the corrective action taking process, the higher the control efficiency and effectiveness. However, the results of these graphs can be further refined to assess the impact of different amount of control budget on the increasing rate (depicted on the top of the figure) of the performance. This figure shows that the increasing rate of the control efficiency and effectiveness exhibits a diminishing trend with more control budget engaged. This behavior is in line with the marginal product theory in economics, as more and more control budget is invested, the increasing rate of control efficiency and effectiveness grows fast initially, when the the reduction in activity duration and the cost invested are not scaled, then at some certain value, the increasing rate starts to go down, while the general performance is increased. Therefore, it is better to explore the optimal point where the increasing rate starts to fall down to make the best use of the limited resources.

\subsection{Combined approach}

In this approach, we propose a fifth combined approach based on the experiments. Table 9 gives a summary of the four methods used before, split up into three components, and also shows the combined approach. This table shows the three components of our study (allocating budget, setting tolerance limits and measuring performance) for the four methods discussed, and also displays a fifth combined method. As discussed in this study, each method makes use of a metric to allocate the limited budget, which can be either the EV, ES or ED for the first three methods, or the SSI for the AR method. Setting up the tolerance limits are all based on either ES or ED. Finally, the performance metrics obviously depends on the metric used for the tolerance limits, i.e. ES has the SPI(t) and ED has the DPI.

In the previous sections, we have shown that the ED approach performs better than the three other approaches for taking corrective actions under limited budget (cf. Fig. 7). Moreover, the experiments also revealed that the AR approach performs slightly better than the ES approach for very serial projects. These two observations have led us to conclude that a combined approach could possibly outperform the ED approach for very serial projects. The 

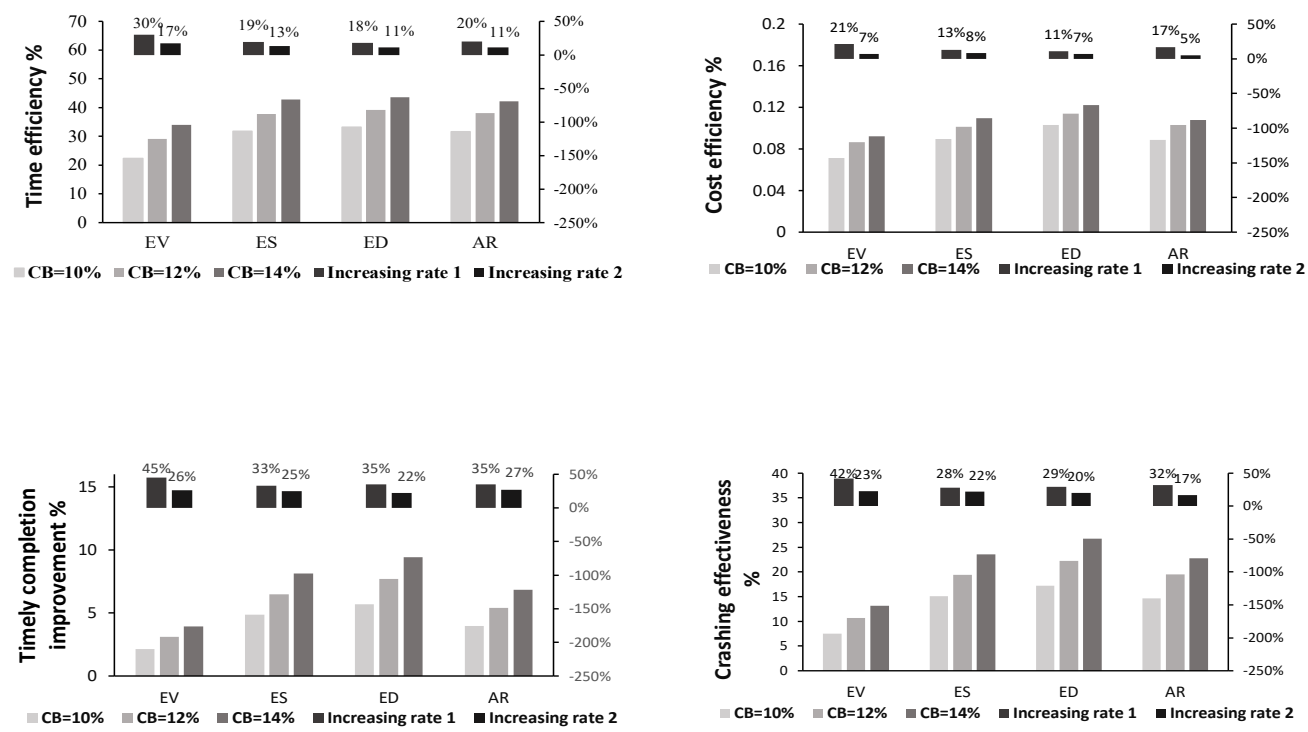

Figure 13: Impact of control budget on performance.

combined approach mixes the overall good performance of the ED approach with some features of the AR approach for very serial projects. More precisely, the combined method relies on the SSI for allocating the budget, but makes use of the ED and DPI metrics for setting tolerance limits and measuring project performance (cf. Table 10).

Fig. 14 compares the performance of this combined method with the best performing ED approach and shows that the combined method further improves all performance metrics for projects wiht SP values higher than 0.8 (serial projects). This behavior is in line with the results of Section 4.3, and is - as mentioned earlier - in contrast with the common knowledge in literature that risk analysis does not perform very well for serial projects.

Table 10: The difference between each approach.

\begin{tabular}{|c|c|c|c|c|c|}
\hline & $\mathrm{EV}$ & $\mathrm{ES}$ & ED & $\mathrm{AR}$ & Combined \\
\hline Allocating budget & EV & ES & ED & SSI & SSI \\
\hline Setting tolerance limits & ES-based & ES-based & ED-based & ES-based & ED-based \\
\hline Measuring performance & $\mathrm{SPI}(\mathrm{t})$ & $\mathrm{SPI}(\mathrm{t})$ & DPI & $\mathrm{SPI}(\mathrm{t})$ & DPI \\
\hline
\end{tabular}

\section{Conclusion}

In this paper, we have investigated the impact of a limited budget for corrective actions on the expected performance of the project. When a project is in progress, corrective actions 

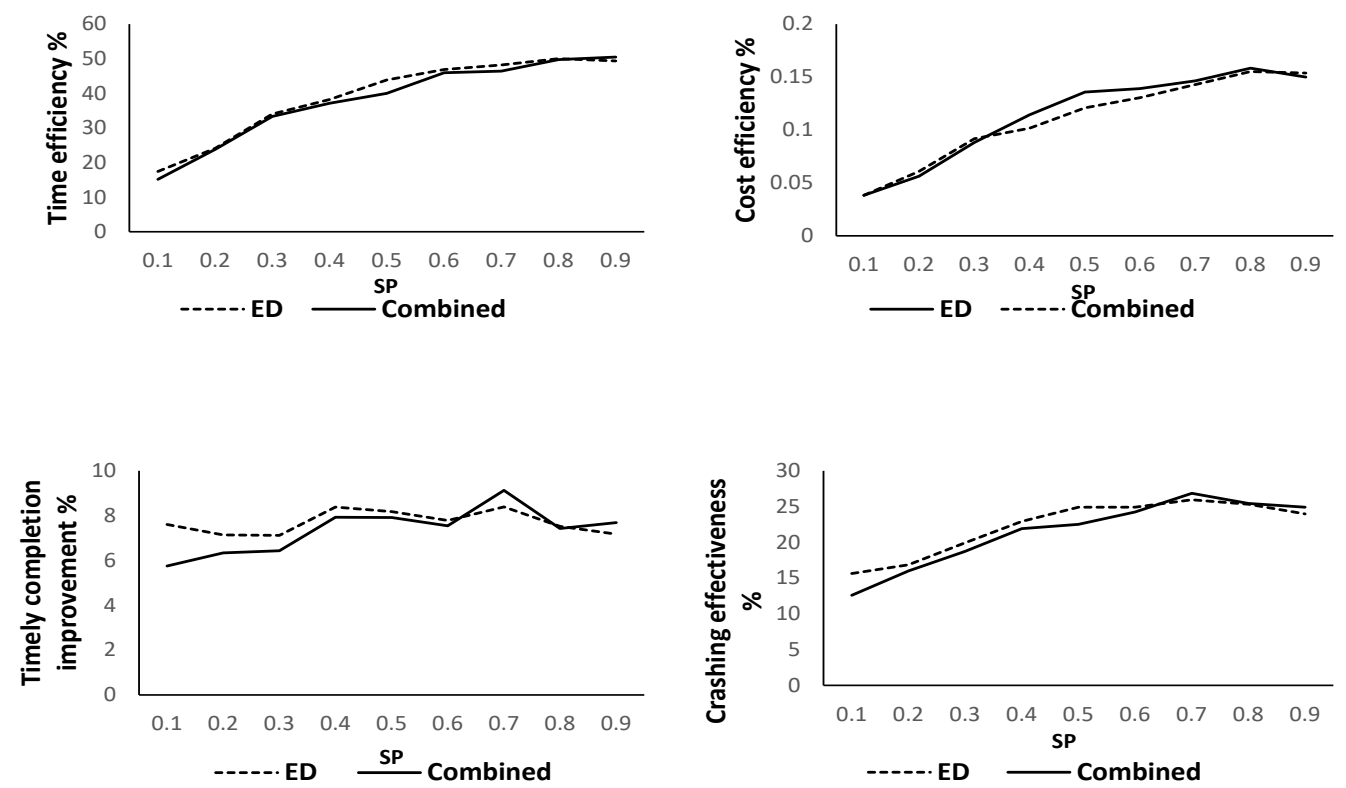

Figure 14: Comparison of the ED and the combined approach.

must be taken whenever the project is expected to be delayed in order to bring the project back on track. Most research studies in the academic literature assume an unlimited budget, however, this is invalid in practice, since the control effort of the project manager is always constrained.

The current research investigates the use of a limited budget to perform corrective actions with the aim of maximizing the expected project outcome. More precisely, four control budget allocation approaches are proposed to determine the amount of allowable budget consumption at each control phase of the project, and different ways of efficiently and effectively take corrective actions to deliver the project on time are analyzed. Three of these four approaches make use of earned value management metrics (EV approach), or extensions to earned schedule (ES approach) or earned duration management (ED approach), while the fourth one relies on metrics measuring activity risk (AR approach). Each of these methods is used in three different versions, labeled as normal version, increasing version and decreasing version. In order to guide this corrective action decision-making process adequately, a large computational experiment is conducted to assess the performance of the comparison of these four approaches, as well as to assess the impact of the project network structure, the impact of using buffers and the size of the corrective action.

First, the computational results show that the ED approach outperforms on average the other three approaches. This ED approach allocates the limited budget according the to earned duration metric, and generates warning signals using tolerance limits set of the duration 
performance index DPI. It has already been shown in Batselier and Vanhoucke (2015) that this ED-DPI approach performs well for predicting the total project duration, but now it shows that it also performs well when taking corrective actions under a limited budget. Hence, our study confirms previous results, and shows that using earned duration management performs well both for project duration forecasting and for taking corrective action to bring projects back on track.

Second, the results also show that the increasing version for each of the four methods always outperforms the other two versions (normal and decreasing) when the budget is limited. This observation is in contrast with the top-down control study of Vanhoucke (2011) who argues that a decreasing attention is better. However, this study did not take a limited budget into account, and simply assumed that actions are not restricted at any time. Consequently, the way top-down control should be optimized depends on the availability of the budget for taking actions.

Third, we have shown that the ES approach and the AR approach both make use of the ES-based tolerance limits and schedule performance index SPI(t) measuring the project performance, and only differ in how to allocate the control budget (one uses the ES while the other uses SSI). The results have shown that the AR approach performs better than the ES approach for serial projects, while the opposite is true for parallel projects. This is somewhat in contrast to the literature in which it has been shown the ES performs better for serial (known as top-down control) and AR for parallel (known as bottom-up control). Consequently, our study has shown that taking corrective actions under a limited budget is fundamentally different from most studies in literature who assume project control without any restrictions.

Based on the observation, we have proposed a combined approach by mixing parts of the ED approach with the AR approach. This combined approach makes use of the SSI to allocate budgets (similar to the AR approach) but set ED-based tolerance limits and measure the project performance using the DPI metric (as in the ED approach). We have shown that such a combined approach performs better than ED and AR individually for very serial projects.

Finally, our results have shown that taking small but frequent actions outperforms a process where significant actions are taken after long periods of project progress.

Our future research topics are twofold. First, the proposed approaches should be implemented and tested on real-life projects to validate our proposed approach, and to find ways to further improve our findings from the current research. Such an approach is promising, but not always easy, since finding enough information about corrective actions is not always practically possible. Second, the budget allocation models of this paper should be extended by taking 
the limited availability of the renewable resources into account. Such an extension will have major implications on the accuracy of the reliability of the used metrics, especially the SSI metric used in the AR approach. Nevertheless, since projects are almost always performed under limited resources, such an extension would create additional insights in how to best allocate a limited budget for project control. 


\section{References}

Acebes, F., Pajares, J., Galán, J. M., and López-Paredes, A. (2014). A new approach for project control under uncertainty. going back to the basics. International Journal of Project Management, 32(3):423-434.

Aliverdi, R., Moslemi Naeni, L., and Salehipour, A. (2013). Monitoring project duration and cost in a construction project by applying statistical quality control charts. International Journal of Project Management, 31(3):411-423.

Ballesteros-Pérez, P., Cerezo-Narváez, A., Otero-Mateo, M., Pastor-Fernández, A., and Vanhoucke, M. (2019). Performance comparison of activity sensitivity metrics in schedule risk analysis. Automation in Construction, 106:102906.

Barraza, G. A. and Bueno, R. A. (2007). Probabilistic control of project performance using control limit curves. Journal of Construction Engineering and Management, 133(4):957-965.

Batselier, J. and Vanhoucke, M. (2015). Evaluation of deterministic state-of-the-art forecasting approaches for project duration based on earned value management. International Journal of Project Management, 33(7):1558-1596.

Bauch, G. T. and Chung, C. A. (2001). A statistical project control tool for engineering managers. Project Management Journal, 32:37-44.

Bie, L., Cui, N., and Zhang, X. (2012). Buffer sizing approach with dependence assumption between activities in critical chain scheduling. International Journal of Production Research, 50(24):7343-7356.

Book, S. (2006). "Earned Schedule" and its possible unreliability as an indicator. The Measurable News, Spring:24-30.

Bowman, R. A. (2006). Developing activity duration specification limits for effective project control. European Journal of Operational Research, 174(2):1191-1204.

Chen, Z., Demeulemeester, E., Bai, S., and Guo, Y. (2019). A bayesian approach to set the tolerance limits for a statistical project control method. International Journal of Production Research, 69:10.1080/00207543.2019.1630766.

Colin, J., Martens, A., Vanhoucke, M., and Wauters, M. (2015). A multivariate approach for top-down project control using earned value management. Decision Support Systems, 79:65-76.

Colin, J. and Vanhoucke, M. (2014). Setting tolerance limits for statistical project control using earned value management. Omega The International Journal of Management Science, 49:107-122.

Colin, J. and Vanhoucke, M. (2015). Developing a framework for statistical process control approaches in project management. International Journal of Project Management, 33:1289-1300.

Demeulemeester, E., Vanhoucke, M., and Herroelen, W. (2003). Rangen: A random network generator for activity-on-the-node networks. Journal of Scheduling, 6:17-38.

Elshaer, R. (2013). Impact of sensitivity information on the prediction of project's duration using earned schedule method. International Journal of Project Management, 31:579-588.

Fleming, Q. and Koppelman, J. (2010). Earned Value Project Management. Project Management Institute, Newton Square, Pennsylvania, 3rd edition edition. 
Hadian, H. and Rahimifard, A. (2019). Multivariate statistical control chart and process capability indices for simultaneous monitoring of project duration and cost. Computers $\&$ Industrial Engineering, pages 788-797.

Hammad, M., Abbasi, A., and Ryan, M. (2016). Developing a novel framework to manage schedule contingency using theory of constraints and earned schedule method. Journal of Construction Engineering and Management, 144(4):04018011.

Hegazy, T. and Petzold, K. (2003). Genetic optimization for dynamic project control. Journal of Construction Engineering and Management, 129(4):396-404.

Hu, X., Cui, N., and Demeulemeester, E. (2014). Effective expediting to improve project due date and cost performance through buffer management. International Journal of Production Research, 53(5):1460-1471.

Hu, X., Cui, N., Demeulemeester, E., and Bie, L. (2016). Incorporation of activity sensitivity measures into buffer management to manage project schedule risk. European Journal of Operational Research, 249:717727.

Khamooshi, H. and Golafshani, H. (2014). EDM: Earned duration management, a new approach to schedule performance management and measurement. International Journal of Project Management, 32(6):10191041.

Kim, B. and Kim, H. (2014). Sensitivity of earned value schedule forecasting to s-curve patterns. Journal of Construction Engineering and Management, 7:04014023.

Kim, T. (2015). Dynamic control thresholds for consistent earned value analysis and reliable early warning. Journal of Management in Engineering, 31(5):04014077.

Kotiah, T. and Wallace, N. D. (1973). Another look at the pert assumptions. Management Science, 20(1):4449 .

Leach, L. P. (2005). Critical chain project management, volume 2nd. Artech House.

Leu, S. S. and Lin, Y. C. (2008). Project performance evaluation based on statistical process control techniques. Journal of Construction Engineering and Management, 134:813-819.

Lipke, W. (2003). Schedule is different. The Measurable News, Summer:31-34.

Lipke, W., Zwikael, O., Henderson, K., and Anbari, F. (2009). Prediction of project outcome: The application of statistical methods to earned value management and earned schedule performance indexes ha ha. International Journal of Project Management, 27:400-407.

Madadi, M. and Iranmanesh, H. (2012). A management oriented approach to reduce a project duration and its risk (variability). European Journal of Operational Research, 219(3):751-761.

Martens, A. and Vanhoucke, M. (2017a). A buffer control method for top-down project control. European Journal Of Operational Research, 262:274-286.

Martens, A. and Vanhoucke, M. (2017b). The integration of constrained resources into top-down project control. Computers $\&$ Industrial Engineering, 110:277-288.

Martens, A. and Vanhoucke, M. (2018). An empirical validation of the performance of project control tolerance limits. Automation in Construction, 89:71-85. 
Martens, A. and Vanhoucke, M. (2019). The impact of applying effort to reduce activity uncertainty on the project time and cost performance. European Journal of Operational Research, 277:442-453.

Ong, H., Wang, C., and Zainon, N. (2016). Integrated earned value gantt chart (ev-gantt) tool for project portfolio planning and monitoring optimization. Engineering Management Journal, 28(1):13-20.

Ou-Yang, C. and Chen, W. (2019). A hybrid approach for project crashing optimization strategy with risk consideration: A case study for an epc project. Mathematical Problems in Engineering, pages 1-17.

Pajares, J. and López-Paredes, A. (2011). An extension of the EVM analysis for project monitoring: The cost control index and the schedule control index. International Journal of Project Management, 29:615-621.

Perkusich, M., Soares, G., Almeida, H., and Perkusich, A. (2016). A procedure to detect problems of processes in software development projects using bayesian networks. Expert Systems with Applications, 46:437-450.

PMBOK (2004). A Guide to the Project Management Body of Knowledge, Third Edition. Newtown Square, Pa.: Project Management Institute, Inc.

Sabeghi, N., Tareghian, H., Demeulemeester, E., and Taheri, H. (2015). Determining the timing of project control points using a facility location model and simulation. Computers \& Operations Research, 61:69-80.

Slowinski, R. (1980). Two approaches to problems of resource allocation among project activities - a comparative study. Journal of Operational Research Society, 8:711-723.

Vanhoucke, M. (2010). Using activity sensitivity and network topology information to monitor project time performance. Omega The International Journal of Management Science, 38:359-370.

Vanhoucke, M. (2011). On the dynamic use of project performance and schedule risk information during project tracking. Omega The International Journal of Management Science, 39:416-426.

Vanhoucke, M. (2012a). Measuring the efficiency of project control using fictitious and empirical project data. International Journal of Project Management, 30:252-263.

Vanhoucke, M. (2012b). Project Management with Dynamic Scheduling: Baseline Scheduling, Risk Analysis and Project Control, volume XVIII. Springer.

Vanhoucke, M. (2018). Tolerance limits for project control: An overview of different approaches. Computers E Industrial Engineering, 127:467-479.

Vanhoucke, M., Coelho, J., Debels, D., Maenhout, B., and Tavares, L. (2008). An evaluation of the adequacy of project network generators with systematically sampled networks. European Journal of Operational Research, 187:511-524.

Vanhoucke, M. and Vandevoorde, S. (2007). A simulation and evaluation of earned value metrics to forecast the project duration. Journal of the Operational Research Society, 58:1361-1374.

Zhang, J., Jia, S., and Diaz, E. (2018). Dynamic monitoring and control of a critical chain project based on phase buffer allocation. Journal of the Operational Research Society, 69:1-12.

Zohoori, B., Verbraeck, A., Bagherpour, M., and Khakdaman, M. (2018). Monitoring production time and cost performance by combining earned value analysis and adaptive fuzzy control. Computers $\mathscr{E}$ Industrial Engineering, pages 805-821. 